\title{
Selfish Random Access over Wireless Channels with Multipacket Reception
}

\author{
Hazer Inaltekin, Member, IEEE, Mung Chiang, Senior Member, IEEE, H. Vincent Poor, Fellow, IEEE, Stephen B. \\ Wicker, Fellow, IEEE
}

\begin{abstract}
This paper analyzes layer 2 contention resolution strategies for wireless networks with multipacket reception by using noncooperative game theory. Necessary and sufficient conditions are obtained for a strategy profile to be a Nash equilibrium. Applications of the derived equilibrium conditions to predict selfish behavior and the resulting equilibrium performance are illustrated in specific communication scenarios along with various design insights. The collective equilibrium behavior of wireless networks with large user populations is also studied, and a Poisson-Bernoulli type approximation is obtained for the total number of packet arrivals. Finally, random access control with imperfect information structure is considered, the form of equilibrium strategies as well as uniqueness and existence results for general wireless channel models are obtained, and the best-response learning dynamics achieving an equilibrium are illustrated in specific instances.
\end{abstract}

Index Terms-Slotted ALOHA, multipacket reception, game theory, contention resolution, medium access control

\section{INTRODUCTION}

$\mathbf{F}$ ourth generation $(4 \mathrm{G})$ wireless communication networks empowered with a new generation of open source operating systems are expected to transform mobile handsets into intelligent terminals for applications such as high-definition video streaming, online gaming, etc. [1]. An emerging vital design challenge in these networks is the distributed coordination and control of increasingly autonomous and heterogenous mobile terminals for efficient allocation of limited communication resources such as bandwidth and power. Game theory and the related field of mechanism design have the potential to guide engineering efforts to overcome such challenges by providing a bottom-up analytical and principled approach to the design of local operation rules and to the verification of resulting collective network behavior through equilibrium analyses.

In particular, recent works [2]-[5] illustrate how game theory provides new insights to reverse/forward engineer existing

Manuscript received January 10, 2011; revised July 13, 2011. Date of current version September 1, 2011. This work was supported in part by the U.S. National Science Foundation under Grant CNS-09-05086, in part by the U.S. Air Force Office of Scientific Research under MURI Grant FA955009-1-0643, and in part by the Australian Research Council under Grant DP11-0102729. The material in this paper was presented in part at the 2nd International ICST Conference on Game Theory for Networks, Shanghai, China, April 2011.

H. Inaltekin is with the Department of Electrical and Electronics Engineering, Antalya International University, Antalya, Turkey. All correspondence should be addressed to H. Inaltekin. (e-mail: hazeri@antalya.edu.tr)

M. Chiang and H. V. Poor are with the Department of Electrical Engineering, Princeton University, Princeton, NJ 08544 USA.

S. B. Wicker is with the School of Electrical and Computer Engineering, Cornell University, Ithaca, NY 14850 USA. medium access control (MAC) protocols, better fairness and service differentiation, higher throughput, and a mechanism by which to decouple contention control from handling failed packets for a class of multiple access networks. Motivated by these recent results, this paper also focuses on layer 2 MAC protocols for next generation wireless networks, and provides new equilibrium results and design insights for such protocols based on noncooperative game theory.

Our main contribution is the derivation of necessary and sufficient Nash equilibrium conditions for setting persistence probabilities in an adaptive $p$-persistent slotted ALOHA protocol in which persistence probabilities are adaptively updated according to the derived equilibrium strategies. These equilibrium conditions are given for general wireless channels with multipacket reception capability. We also provide applications to commonly used channel models to illustrate how the derived equilibrium strategies can be utilized to provide service differentiation and to design user utility functions optimally in multiple access networks. In the second half of the paper, these results are first used to establish a Poisson-Bernoulli type approximation for the limiting distribution of the total number of packet arrivals at Nash equilibrium for large user populations, and are then extended to imperfect information random access games. In the imperfect information case, we observe that a Braess-like paradox occurs and the network throughput is reduced if more information is publicly available about user utility functions.

\section{A. Background and Literature Review}

Wireless channels are inherently broadcast in nature. Therefore, the resolution of contention among mobile users for access to common wireless communication resources remains as a fundamental bottleneck in wireless multiple access communication. In particular, transmissions through wireless channels must be coordinated to control multiple access interference (MAI), and thereby to maximize target data rates. Indeed, this problem has received considerable attention in the literature, and questions such as what packet arrival rates are required to stabilize transmitters' queues and channel throughput are relatively well-understood when all mobile users obey a predetermined set of rules for choosing their transmission probabilities, e.g., see [6]-[10]. Based on these studies as well as many others, various contention based random access approaches have been proposed in [11], including the most prominent ones such as slotted ALOHA, CSMA/CA and IEEE 802.11 DCF, which do not require centralized scheduling. A 
key design degree of freedom in these random access protocols is channel access probability determination, or contention resolution, to mitigate MAI. Different protocols differ in how they implement contention resolution, with prominent examples being window based and persistence probability based approaches.

In addition to the classical approach to contention control, there has been a considerable amount of more recent work such as [2]-[5] and [12]-[20] providing a new game theoretic design perspective for contention resolution. These studies are mainly motivated by ideas revolving around distributed control and coordination of increasingly more heterogenous and programmable wireless communication devices to engineer next generation wireless MAC protocols and technologies. In [2][4] and [12], the main focus is on the dynamics of iterative strategy update mechanisms such as best-response, gradient, or Jacobi play, achieving a desired equilibrium operating point over collision channels. In [12], the authors consider the classical slotted ALOHA setting in which mobile users update their transmission probabilities in a distributed manner to achieve their target throughput levels. This work establishes local convergence properties for the proposed Jacobi strategy update mechanism to a Nash equilibrium operating point.

In [2] and [3], the authors extend the framework introduced in [12] by interpreting contention control as a dynamic iterative feedback system responding to the current contention measure and transmission probabilities of mobile users. This extended model allows for the analysis of maximum network throughput for heterogenous networks with different traffic classes subject to fairness constraints. Among many other results, this work illustrates that the game theoretic approach provides higher throughput, better fairness and service differentiation, and a mechanism to decouple contention control from handling failed packets when compared with the standard IEEE 802.11 DCF. In [4], the authors reverse-engineer the exponential back-off based MAC protocols, and show that the contention resolution protocols implicitly solve a noncooperative random access game. This work provides an explicit expression for user utilities, and studies convergence dynamics for the derived noncooperative random access game. Similar connections are also made for the slotted ALOHA persistence probability in [5].

In addition to the above set of previous works focusing on strategy update mechanisms, there is a large body of papers such as [13]-[18] concentrating more on the fundamental equilibrium properties of the proposed random access game models, including the existence and the uniqueness of Nash equilibria. In [13], the authors propose a simple game theoretic contention resolution approach for a slotted ALOHA type MAC layer over collision channels in which a common utility function parameterized by the cost of transmission arbitrates transmission probabilities of all mobile users. The existence of a symmetric Nash equilibrium at which the throughput performance of the proposed game theoretic MAC layer is almost the same as that of a centrally controlled slotted ALOHA MAC layer is shown.

The model in [13] has been extended in several directions in subsequent works [14]-[18]. In [14], the authors analyze the same model used in [13] over wireless channels with multipacket reception capabilities, and establish the existence of the symmetric Nash equilibrium as well as packet arrival rates stabilizing the queues for some well known channels. In [15], the same game model over collision channels but with heterogenous set of mobile users is investigated. Necessary and sufficient conditions for a strategy profile to be a Nash equilibrium are obtained. The same authors have also extended these results to imperfect information games [16] and to simple multipacket reception models [17]. In [18], the authors generalize the results in [14] to the multipacket reception model using channel state information to schedule transmissions. The existence of a Nash equilibrium operating point is shown, and a stochastic gradient based strategy update mechanism converging to this equilibrium is proposed.

Other game models for random access have also been considered in the literature, e.g., [19] and [20]. In [19], the authors use a Markovian model to express the channel throughput as a function of mobile users' transmission probabilities over collision channels. This model is then used to study the symmetric equilibrium contention resolution strategies in a game setting in which each mobile user aims to maximize its own channel throughput selfishly. Through numerical analysis, it is shown that the overall channel throughput achieved by selfish mobile users coincides with the throughput that can be achieved via centralized controller, as in [13], if a pricing mechanism is employed. In [20], the optimal choice of transmission probabilities subject to a minimum throughput demand over collision channels is considered. In the model employed, channel state information is used to schedule transmissions, similar to the model in [18]. It is shown that there are only two Nash equilibria, one is strictly better than the other one with possibly arbitrarily large performance gap, when the throughput demands are feasible. A strategy update mechanism converging to the better Nash equilibrium operating point is also proposed in this work.

\section{B. Summary of Key Contributions, Comparison with Previous Work and Paper Organization}

1) Summary of Key Contributions: Our contributions in this paper can be summarized in detail as follows.

- We focus on layer 2 contention resolution strategies, and establish new analytically tractable necessary and sufficient conditions for a contention strategy profile to be a Nash equilibrium over wireless channels with multipacket reception capability. Multipacket reception is an important feature of our model to capture probabilistic receptions in wireless multiple access channels. We demonstrate tangible applications of these results in practical communication scenarios, and provide engineering insights to achieve service differentiation and optimal throughput by designing user utility functions. Both perfect information and imperfect information game settings are considered.

- We study the collective equilibrium network behavior for large user populations, and obtain a Poisson-Bernoulli type approximation for the limiting distribution of the total number of packet arrivals at Nash equilibrium, which 
is an important summary statistic to stabilize multiple access networks.

- For the random access game with imperfect information structure, we show that a Nash equilibrium strategy profile, if it exists, must be a threshold strategy profile in which users transmit only if their cost of packet failures is smaller than a threshold value. We provide explicit expressions for setting equilibrium threshold values. We establish existence and uniqueness results in this case, and illustrate learning dynamics on the best-response path to reveal information structure of the game and to learn how to play a Nash equilibrium. In particular, we show that symmetric equilibrium, desirable for fairness purposes, exists but the best-response iteration may never lead to it even in symmetric communication scenarios when there are multiple equilibria.

- This paper shows, for the first time, that a Braess-like paradox occurs if more information is publicly available about user utility functions. That is, the channel throughput achieved under imperfect information turns out to be higher than the one achieved under perfect information. This result hints at an important design criterion for next generation wireless random access protocols: Always maintain some level of randomness to better tame selfishness in mobile users for higher target data rates.

2) Comparison with Previous Work: These results improve the current state-of-the-art in game theory based random access design and analysis in several important ways. Unlike the works focusing on iterative strategy update mechanisms and transient network behavior such as [2]-[4] and [12], we establish necessary and sufficient conditions to be satisfied by steady-state equilibrium contention resolution strategies (see Section III). These equilibrium conditions can be solved either analytically or numerically to obtain equilibrium transmission probabilities (see Section IV), and then the network can be readily stabilized to a desired equilibrium by broadcasting these probabilities to mobile users. For the imperfect information game formulation, even though the existence and uniqueness of the equilibrium can be established, such closedform or numerical solutions are not readily available, and therefore the transient network behavior is also investigated by studying learning dynamics on the best-response path (see Section VI).

In comparison to works focusing on random access only over collision channels such as [12], [13], [15], [16], [19] and [20], the physical layer model considered in this paper is more general, including the collision channel model as a special case. In addition, we consider both symmetric and asymmetric equilibrium strategies, e.g., see the first example in Section IV, rather than focusing only on symmetric contention resolution strategies as in [13] and [19]. Analysis of asymmetric equilibria is especially important for understanding how to provision differentiated services to different classes of users in increasingly heterogenous wireless networks. Similar to our problem formulation, multipacket reception capability in the random access setting is also considered in [14] and [18]. As compared to the existence results, which provide limited infor- mation about the structure of equilibrium strategies, appearing in these works, we obtain detailed necessary and sufficient equilibrium conditions, and solve them, either analytically or numerically, to derive the shape of equilibrium contention resolution strategies. In addition, neither the asymptotic collective network behavior as in Section $\mathrm{V}$ nor the game formulations with imperfect information as in Section VI are considered in [14] and [18].

3) Paper Organization: The rest of the paper is organized as follows. Section II describes the system model under consideration. Equilibrium transmission strategies for general wireless channels with multipacket reception capability are analyzed in Section III, and then applications of these results along with various engineering insights are illustrated in Section IV. Section V studies the collective equilibrium network behavior for a large user population. Section VI investigates possible extensions of the basic model to imperfect information random access games. Finally, Section VII concludes the paper.

\section{SySTEM MODEL}

We consider a wireless multiple access communication network in which $N$ mobile users, indexed by $\mathcal{I}=\{1, \ldots, N\}$, are selfishly contending for channel access to communicate with a common base station (BS). The time is slotted, and packet transmissions are synchronized at the beginning of each time slot. The physical layer of the system is modeled by a stochastic channel reception matrix $\boldsymbol{R}=\left(r_{n, k}\right)_{\substack{1 \leq n \leq N \\ 0 \leq k \leq N}}$, where $r_{n, k}$ represents the probability that $k$ packets are received correctly given $n$ of them are transmitted. By convention, $r_{n, k}$ is set to zero if $k>n$. Note that $\sum_{k=0}^{N} r_{n, k}=1$ for all $n \in\{1, \ldots, N\}$. If $r_{1,0}>0$, we say that the channel is imperfect (or, noisy) due to various random factors such as fading, path-loss and background noise.

We assume that all users involved in a collision of size $n$ have identical packet success probabilities, which are given by $\gamma_{n}=\frac{1}{n} \sum_{k=1}^{n} k r_{n, k}$. In such a multiple access setting, the success probability of a packet is expected to decrease with increasing MAI. In this paper, we model this downward trend by assuming $\gamma_{n+1} \leq \gamma_{n}$. In order to avoid trivialities, we also assume that $\gamma_{n+1}$ is strictly smaller than $\gamma_{n}$ for at least one $n$ in $\{1, \ldots, N-1\}$. Otherwise, packet failures do not depend on the interference caused by other users, and the resulting communication scenario is easy to analyze. The set of such stochastic channel matrices will be represented by $\mathcal{R}$.

If mobile user $i$ transmits a packet successfully, it receives a utility of $u_{i, S}$ units. If the transmission fails, it receives a utility of $u_{i, F}$ units. If it waits, it receives a utility of $u_{i, W}$ units. We assume that $u_{i, S}>u_{i, W}>u_{i, F}$, and use the vector notation $\boldsymbol{u}_{i}=\left(u_{i, S}, u_{i, W}, u_{i, F}\right)$. These utilities are needed for setting up a selfish random access utility maximization problem for the multiple access communication scenario under consideration, as well as for allowing us to model different battery levels, delay and quality-of-service requirements of different users. The random access game $\mathcal{G}$ is defined to be the triple $\mathcal{G}=\left\langle\mathcal{I},\left\{\mathcal{S}_{i}\right\}_{i \in \mathcal{I}},\left\{\boldsymbol{u}_{i}\right\}_{i \in \mathcal{I}}\right\rangle$, where $\mathcal{S}_{i}=\left[\alpha_{i}, \beta_{i}\right] \subseteq[0,1]$ is the set from which user $i$ chooses a 
transmission probability (i.e., a contention resolution strategy) to access the wireless channel. Here, $\alpha_{i}$ and $\beta_{i}$ are positive real numbers satisfying $0 \leq \alpha_{i} \leq \beta_{i} \leq 1, \forall i \in \mathcal{I}$. We will interpret contention resolution strategies as persistence probabilities in an adaptive $p$-persistent slotted ALOHA protocol in which persistence probabilities are adaptively updated according to the derived equilibrium strategies. Persistence probability based contention resolution can also approximate a contention window based protocol [4].

If the strategy sets $\mathcal{S}_{i}$ and utility vectors $\boldsymbol{u}_{i}$ of all users are identical, we say $\mathcal{G}$ is a symmetric random access game. In this case, the common strategy set from which all users choose their transmission probabilities is given by $[\alpha, \beta]$, where $\alpha$ and $\beta$ are positive real numbers satisfying $0 \leq \alpha \leq \beta \leq 1$. We say $\mathcal{G}$ is in the standard form if utility vectors are in the form of $\boldsymbol{u}_{i}=\left(1,0,-c_{i}\right)$, where $c_{i}>0$ is interpreted as the cost of packet failure for user $i$.

The average utility that a user receives is a function of its transmission probability and the transmission probabilities of other users. Let $\mathcal{S}=\prod_{i \in \mathcal{I}} \mathcal{S}_{i}$ be the product set of user strategies, $\boldsymbol{p}$ be a vector of transmission probabilities in $\mathcal{S}$, and $U_{i}(\boldsymbol{p})$ be the expected utility that the $i$ th user receives as a function of $\boldsymbol{p}$. The selfish optimization problem to be solved by user $i$ is to find $p_{i}^{\star}$ such that $U_{i}\left(p_{i}^{\star}, \boldsymbol{p}_{-i}\right) \geq U_{i}\left(p_{i}, \boldsymbol{p}_{-i}\right)$ for all $p_{i} \in \mathcal{S}_{i}$, where $\boldsymbol{p}_{-i}$ represents the vector of transmission probabilities of the other users. We say that $\boldsymbol{p}^{\star}=\left(p_{i}^{\star}\right)_{i \in \mathcal{I}}$ is a Nash equilibrium if and only if $U_{i}\left(p_{i}^{\star}, \boldsymbol{p}_{-i}^{\star}\right) \geq U_{i}\left(p_{i}, \boldsymbol{p}_{-i}^{\star}\right)$ for all $i \in \mathcal{I}$ and $p_{i} \in \mathcal{S}_{i}$. Let $\mathcal{N}_{\mathcal{G}}(\boldsymbol{R})$ denote the set of Nash equilibria of $\mathcal{G}$ when the channel reception matrix is $\boldsymbol{R}$. We say that two random access games $\mathcal{G}_{j}, j \in\{1,2\}$, are equivalent if $\mathcal{N}_{\mathcal{G}_{1}}(\boldsymbol{R})=\mathcal{N}_{\mathcal{G}_{2}}(\boldsymbol{R})$ for all $\boldsymbol{R} \in \mathcal{R}$.

\section{Equilibrium Strategies FOR GENERAL WiRELESS CHANNELS}

In this section, we analyze equilibrium contention resolution strategies for general wireless channels and obtain necessary and sufficient conditions to be satisfied by a Nash equilibrium transmission probability vector. In the next section, we will illustrate the applications of our results in more specific communication scenarios by solving these equilibrium conditions.

The first critical issue to resolve is the existence of a Nash equilibrium. To this end, a positive existence result directly follows from the Glicksberg fixed point theorem [21]. (See also Theorem 1.2 in [22].) However, such existence results provide limited information about the structure of the Nash equilibria, and about selfish transmission probabilities at these equilibria. Therefore, we establish necessary and sufficient conditions to be satisfied by a Nash equilibrium transmission probability vector in the following theorem. We will then solve these conditions to obtain explicit representations of equilibrium contention resolution strategies in some special cases.

Theorem 1: For a given channel reception matrix $\boldsymbol{R}$ and a strategy profile $\boldsymbol{p}$, let

$$
\Gamma_{i}\left(\boldsymbol{R}, \boldsymbol{p}_{-i}\right)=\sum_{n=1}^{N} \sum_{\substack{\mathcal{I}_{n} \subseteq \mathcal{I}: \\ i \in \mathcal{I}_{n},\left|\mathcal{I}_{n}\right|=n}} \gamma_{n} \prod_{j \in \mathcal{I}_{n}-\{i\}} p_{j} \prod_{j \in \mathcal{I}-\mathcal{I}_{n}}\left(1-p_{j}\right) .
$$

Then, $\boldsymbol{p}^{\star}$ is a Nash equilibrium if and only if the following equilibrium conditions hold for all $i \in \mathcal{I}$ :

(i) If $\Gamma_{i}\left(\boldsymbol{R}, \boldsymbol{p}_{-i}^{\star}\right)>\frac{u_{i, W}-u_{i, F}}{u_{i, S}-u_{i, F}}$, then $p_{i}^{\star}=\beta_{i}$.

(ii) If $\Gamma_{i}\left(\boldsymbol{R}, \boldsymbol{p}_{-i}^{\star}\right)=\frac{u_{i, W}-u_{i, F}}{u_{i, S}-u_{i, F}}$, then $\alpha_{i} \leq p_{i}^{\star} \leq \beta_{i}$.

(iii) If $\Gamma_{i}\left(\boldsymbol{R}, \boldsymbol{p}_{-i}^{\star}\right)<\frac{u_{i, W}-u_{i, F}}{u_{i, S}-u_{i, F}}$, then $p_{i}^{\star}=\alpha_{i}$.

Proof: See Appendix A.

Intuitively, the terms appearing on the left-hand sides of these equilibrium conditions can be considered to be the contention signal that each mobile user receives from other users in the network. A higher contention signal received by user $i$ means less MAI is generated by other users, and therefore the higher the channel access and the resulting packet success probabilities of user $i$ are.

In Section IV, we show that these equilibrium conditions can be solved to derive the form of equilibrium transmission probabilities for specific channel models. Once these probabilities are derived, two engineering approaches are possible. In the first one, a Stackelberg formulation can be considered in which the BS moves first, and dictates the transmission probabilities corresponding to a desired equilibrium point. In the second approach, we can design a distributed strategy update mechanism, possibly based on a Lyapunov function as in [4] and [12], to lead the network to a desired equilibrium. We will illustrate one such possible strategy update mechanism in Section VI. Another important consequence of this theorem is a representation of random access games in terms of equivalence classes.

Proposition 1: Any random access game $\mathcal{G}=$ $\left\langle\mathcal{I},\left\{\mathcal{S}_{i}\right\}_{i \in \mathcal{I}},\left\{\boldsymbol{u}_{i}\right\}_{i \in \mathcal{I}}\right\rangle$ is equivalent to the standard form random access game $\mathcal{G}^{\prime}=\left\langle\mathcal{I},\left\{\mathcal{S}_{i}\right\}_{i \in \mathcal{I}},\left\{\boldsymbol{u}_{i}^{\prime}\right\}_{i \in \mathcal{I}}\right\rangle$ with $c_{i}=\frac{u_{i, W}-u_{i, F}}{u_{i, S}-u_{i, W}}$.

Proof: The proof follows directly from Theorem 1 by observing that $\frac{c_{i}}{1+c_{i}}=\frac{u_{i, W}-u_{i, F}}{u_{i, S}-u_{i, F}}$.

Therefore, without loss of any generality, we will focus only on random access games in the standard form in the rest of the paper. Technically speaking, standard form random access games will be used to represent equivalence classes of random access games, and analyzing the Nash equilibria of a standard form game will amount to analyzing the Nash equilibria of random access games in the same equivalence class. In the next theorem, we establish a symmetry property for equilibrium contention resolution strategies in symmetric random access games.

Theorem 2: Let $\mathcal{G}$ be a symmetric random access game in standard form with the common strategy set $[\alpha, \beta] \subseteq[0,1]$ and the common cost of packet failure $c>0$ for all users. If $\boldsymbol{p}^{\star}$ is a Nash equilibrium, then $p_{i}^{\star}=p_{j}^{\star}$ for all $p_{i}^{\star}$ and $p_{j}^{\star}$ in $(\alpha, \beta)$. In particular, if $p_{i}^{\star} \in(\alpha, \beta)$ for all $i \in \mathcal{I}$, then all users access the channel with the same transmission probability $p^{\star}$ solving

$$
J\left(p^{\star}\right)=\frac{c}{1+c},
$$

where $J(p)=\sum_{n=0}^{N-1} \gamma_{n+1}\left(\begin{array}{c}N-1 \\ n\end{array}\right) p^{n}(1-p)^{N-1-n}$. Proof: See Appendix B.

We note that (1) is also sufficient for $p^{\star}$ to be the common equilibrium transmission probability for all users, as implied 


$$
\frac{d J(p)}{d p}=-\sum_{n=1}^{N-1}\left(\gamma_{n}-\gamma_{n+1}\right) \frac{(N-1) !}{(n-1) !(N-1-n) !} p^{n-1}(1-p)^{N-1-n}<0
$$

by Theorem 1. Moreover, the solution to (1), if it exists, is unique. This can be readily seen by taking the derivative of $J(p)$ with respect to $p$ as in (2) Finally, a necessary and sufficient condition for the existence of the solution to (1) is to have $J(\alpha) \geq \frac{c}{1+c} \geq J(\beta)$.

\section{Applications And Discussion}

We will now demonstrate tangible applications of the above general equilibrium results in more specific communication scenarios. We start our discussion with equilibrium contention resolution strategies for imperfect collision channels.

\section{A. Selfish Random Access over Imperfect Collision Channels}

One of the most commonly studied channel models for multiple access communication is the classical collision channel model, perhaps mainly due to its analytical tractability for obtaining practical control mechanisms achieving the maximum stable throughput of such systems, e.g., see [7], [8] and [11] for more details. We now look briefly at equilibrium contention resolution strategies for imperfect wireless collision channels. The derived Nash equilibrium strategies can, in turn, be used in a drift analysis to determine the stability of the resulting Markovian communication system with selfish mobile users. However, we will not pursue this direction in the current paper.

1) Channel Model: In the collision channel model, a packet transmission is assumed to be successful only if there is no other user attempting to transmit simultaneously. Hence, $r_{n, k}=\delta_{0, k}$ if $n \geq 2$, where $\delta_{i, j}=1$ if $i=j$, and zero otherwise. We let $r_{1,0}=\theta$ and $r_{1,1}=1-\theta$ for some $\theta \in[0,1]$. Here, the parameter $\theta$ can be interpreted as a measure of the noise level summarizing all random factors such as background noise, fading and path-loss affecting packet receptions. The smaller the $\theta$ is, the less noise is present in the system, and a packet transmission is more likely to be successful if there is no other transmission attempt. On the other hand, if $\theta$ is large, it is more likely that a packet fails even if there is no other user transmitting simultaneously.

2) Equilibrium Contention Resolution Strategies: By setting the strategy sets to $[0,1]$, we can simplify the equilibrium conditions in Theorem 1 as follows: $\boldsymbol{p}^{\star}$ is a Nash equilibrium if and only if, for all $i \in \mathcal{I}$, it satisfies (i) $p_{i}^{\star}=1$ if $(1-\theta) \prod_{j \neq i}\left(1-p_{j}^{\star}\right)>\frac{c_{i}}{1+c_{i}}$, (ii) $p_{i}^{\star} \in[0,1]$ if $(1-\theta) \prod_{j \neq i}\left(1-p_{j}^{\star}\right)=\frac{c_{i}}{1+c_{i}}$, and (iii) $p_{i}^{\star}=0$ if $(1-\theta) \prod_{j \neq i}\left(1-p_{j}^{\star}\right)<\frac{c_{i}}{1+c_{i}}$.

We can further simplify these conditions by dividing users into three disjoint groups given by $\mathcal{I}^{1}=\left\{i \in \mathcal{I}: c_{i}>\frac{1-\theta}{\theta}\right\}$, $\mathcal{I}^{2}=\left\{i \in \mathcal{I}: c_{i}=\frac{1-\theta}{\theta}\right\}$ and $\mathcal{I}^{3}=\left\{i \in \mathcal{I}: c_{i}<\frac{1-\theta}{\theta}\right\}$. Users in $\mathcal{I}^{1}$ have costs so large that they never transmit even if no other user is contending for channel access. If $\mathcal{I}^{1}=\mathcal{I}$, the only Nash equilibrium is the one at which none of the users transmits, which never arises as a Nash equilibrium when the channel is noise-free, i.e., $\theta=0$. A user in $\mathcal{I}^{2}$ transmits with positive probability only if all other users set their transmission probabilities to zero. More precisely, a transmission probability vector $\boldsymbol{p}^{\star}$ at which there exists a user $i \in \mathcal{I}^{2}$ transmitting with a probability $p_{i}^{\star} \in\left[1-\frac{1}{1-\theta} \frac{\eta}{1+\eta}, 1\right]$, and $p_{j}^{\star}=0$ for all other $j \neq i$ is a Nash equilibrium of $\mathcal{G}$, where $\eta=\min _{j \in \mathcal{I}^{3}} c_{j}$. The lower bound $1-\frac{1}{1-\theta} \frac{\eta}{1+\eta}$ on $p_{i}^{\star}$ is chosen such that all users in $\mathcal{I}^{3}$ are deterred from transmission, and it is easy to show that this lower bound is always positive.

There are interesting Nash equilibria at which a subset $\mathcal{I}^{3,0}$ of users in $\mathcal{I}^{3}$ transmit with positive probability, while others exercise zero transmission probability as their contention resolution strategies. Let $\boldsymbol{p}^{\star}$ be such a Nash equilibrium. Then, contention resolution strategies of users in $\mathcal{I}^{3,0}$ must satisfy the second equilibrium condition, which leads to closed form expressions $p_{i}^{\star}=1-\frac{1+c_{i}}{c_{i}}\left(\frac{1}{1-\theta} \varphi\left(\mathcal{I}^{3,0}\right)\right)^{\overline{\mathcal{I}}^{3,0} \mid-1}, \forall i \in \mathcal{I}^{3,0}$, where the set function $\varphi: 2^{\mathcal{I}^{3}}-\{\emptyset\} \mapsto \mathbb{R}_{+}$is defined as $\varphi\left(\mathcal{I}^{3,0}\right)=\prod_{i \in \mathcal{I}^{3,0},} \frac{c_{i}}{1+c_{i}}$ for all non-empty subsets $\mathcal{I}^{3,0}$ of $\mathcal{I}^{3}$. (Here, $2^{\mathcal{I}^{3}}$ denote the set of all subsets of $\mathcal{I}^{3}$.) Note that such a solution is feasible only if $\left(\frac{1}{1-\theta} \varphi\left(\mathcal{I}^{3,0}\right)\right)^{\frac{1}{\mathcal{I}^{3,0} \mid-1}} \leq$ $\frac{c_{i}}{1+c_{i}}<1$, which further implies $\varphi\left(\mathcal{I}^{3,0}\right)<1-\theta$. Therefore, when $\left|\mathcal{I}^{3,0}\right|=1$, we set $p_{i}^{\star}$ to 1 for $i \in \mathcal{I}^{3,0}$ without causing any ambiguity. These transmission probabilities also show how different services are provisioned to different users belonging to different traffic classes at the equilibrium. For example, by interpreting $c_{i}$ and $p_{i}^{\star}$ as the common delay price (arising from packet collisions) and the channel access probability for class- $i$ users, respectively, we expect users carrying more timesensitive services such as real-time video streaming to have larger delay prices, and access the channel more frequently. Indeed, $\frac{1-p_{i}^{\star}}{1-p_{j}^{\star}}=\frac{\left(1+c_{i}\right) c_{j}}{\left(1+c_{j}\right) c_{i}}<1$ for $c_{i}>c_{j}$.

3) Numerical Results and Discussion: In Fig. 1, we illustrate equilibrium strategies on different partitions of $\mathbb{R}_{+}^{2}$ for the two-user communication scenario. For $N>2$, we can conceptually visualize a similar partition of $\mathbb{R}_{+}^{N}$ into $2^{N}$ box-like regions. In particular, if all $c_{i}$ 's are small, i.e., $0<c_{i}<\frac{1-\theta}{\theta}$ for all $i \in \mathcal{I}$, then the users access the channel with positive probability. On the other hand, as the $c_{i}$ 's increase above the critical level $\frac{1-\theta}{\theta}$, users start to switch off by setting their transmission probabilities to zero. If the channel quality or the costs of failed packets are changing over time, this behavior is reminiscent of an opportunistic random access policy in which selfish users access the channel opportunistically when the channel quality is above a threshold determined by the costs of packet failures, i.e., when $1-\theta>\frac{c_{i}}{1+c_{i}}$ for user $i$. In another interpretation, we can say that when the channel is in a deep fade, the users with high costs cannot be provisioned for the required level of quality-of-service regardless of the amount of contention from other users, and therefore traffic from them is completely blocked and all communication resources are 


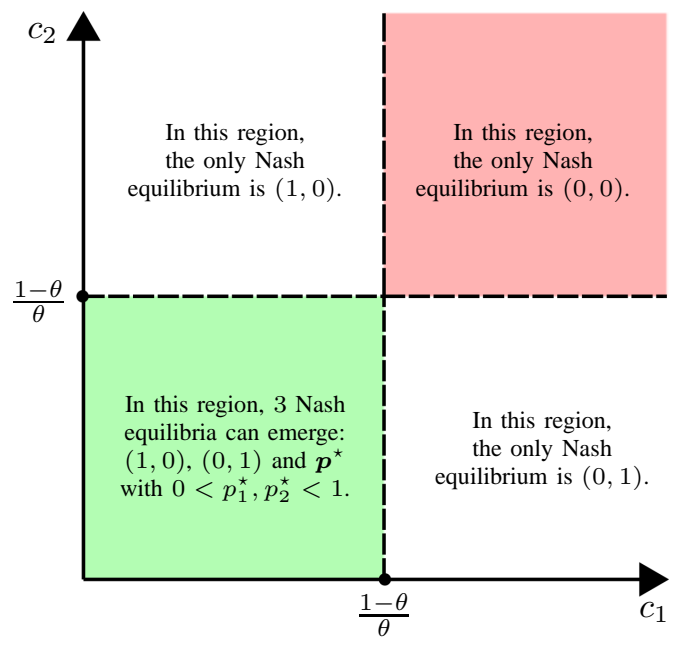

Fig. 1. Possible equilibrium contention resolution strategies on different partitions of $\mathbb{R}_{+}^{2}$ for the imperfect collision channel with two users. $p_{i}^{\star}=$ $1-\frac{1}{1-\theta} \frac{c_{-i}}{1+c_{-i}}$, where $i,-i \in\{1,2\}$ and $i \neq-i$.

distributed among other users with low costs until the channel recovers from the deep fade.

In Fig. 2, we plot the equilibrium transmission probabilities for the homogenous case versus $c$ by setting $N$ to 5 . We focus only on the equilibrium at which all users transmit with the same positive probability, which corresponds to the fair allocation of communication resources. The equilibrium transmission probability is, then, given by $p^{\star}=1-\sqrt[N-1]{\frac{1}{1-\theta} \frac{c}{1+c}}$. As expected, when the noise level $\theta$ increases, transmission probabilities and the system throughput measured in packets per time slot decrease. In all cases, small values of $c$ lead to high transmission probabilities, which in turn results in excessive packet collisions and low throughput. Similarly, large values of $c$ result in channel under-utilization, and therefore low throughput. In the middle-ground, there exists an optimal level of $c$ maximizing the system throughput. It is easy to see that this maximum throughput is also the best that we can achieve via a central controller since transmission probabilities are continuous functions of costs. Therefore, no loss is incurred by selfish operation if selfish transmission probabilities can be manipulated to drive the system to the optimal operating point.

For example, Fig. 2 suggests that when $c$ is small, a central controller can use the parameter $\theta$ as a signaling device to manipulate transmission probabilities, and drive the system to the optimal operating point, either by declaring a fictitious noise level to be greater than the true noise level, or by introducing artificial noise during the channel estimation phase. This approach will decrease users' greediness, eliminate excessive collisions and increase the system throughput. This operation can also be considered as a design process for user utility functions based on changing the effective value of $c$ to achieve optimal performance. Figure 3 illustrates that the throughput increases significantly, and the same maximum throughput can be achieved by declaring fictitious noise levels $0.8,0.5$ and 0.2 when $c$ is around $0.1,0.27$ and 0.5 , respectively, for a noisefree channel.
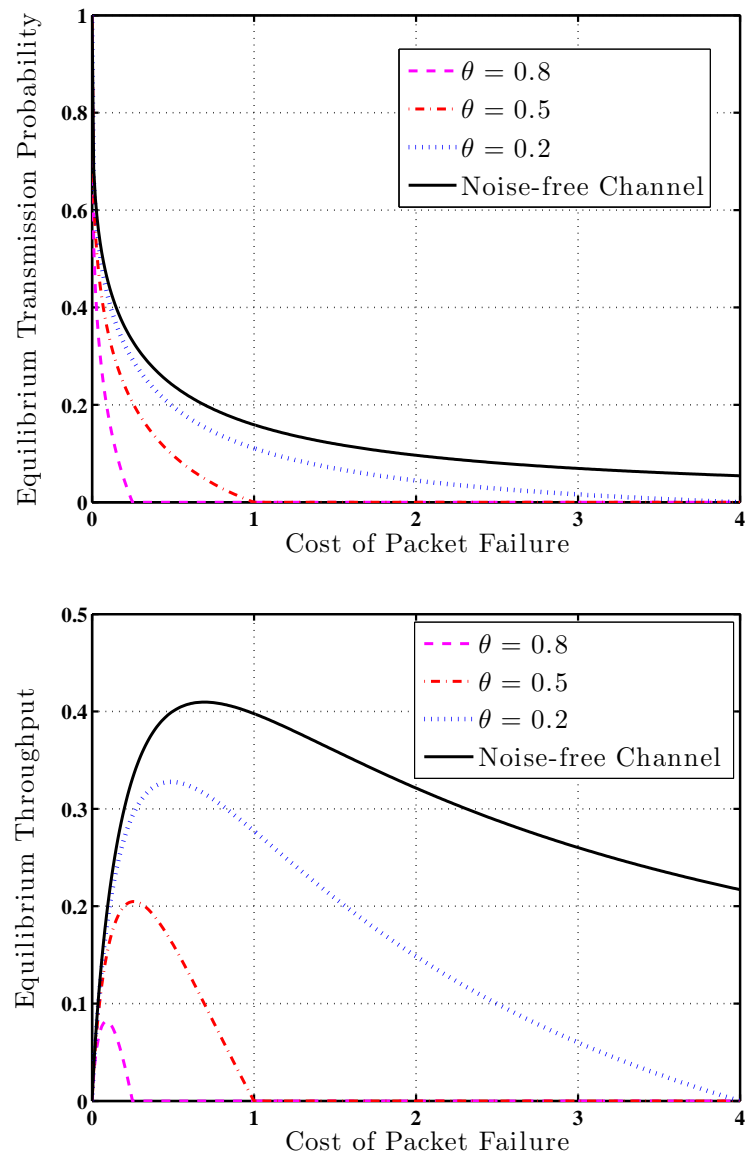

Fig. 2. Equilibrium contention resolution strategies (top figure) and the corresponding system throughput (bottom figure). Imperfect collision channels with $N=5$. The unit of throughput is normalized to packets per time slot.

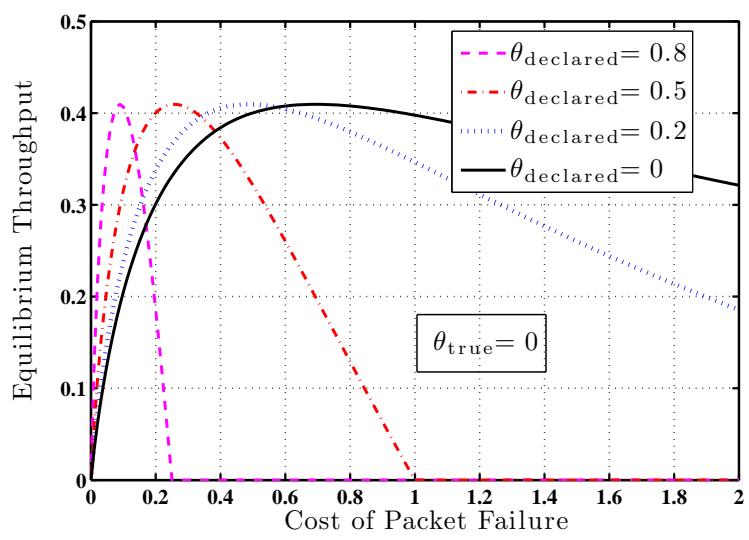

Fig. 3. System throughput when selfish users are manipulated by declaring fictitious noise levels. Perfect collision channel with $N=5$. The unit of throughput is normalized to packets per time slot.

\section{B. Selfish Random Access for T-out-of-N Channels}

1) Channel Model: The second application of our results will be to a special type of multipacket reception channel in which all packets can be reconstructed successfully with probability $1-\theta_{n}$ if the collision size $n$ is smaller than or equal to $T \in\{1,2, \cdots, N\}$. On the other hand, if $n>T$, all 

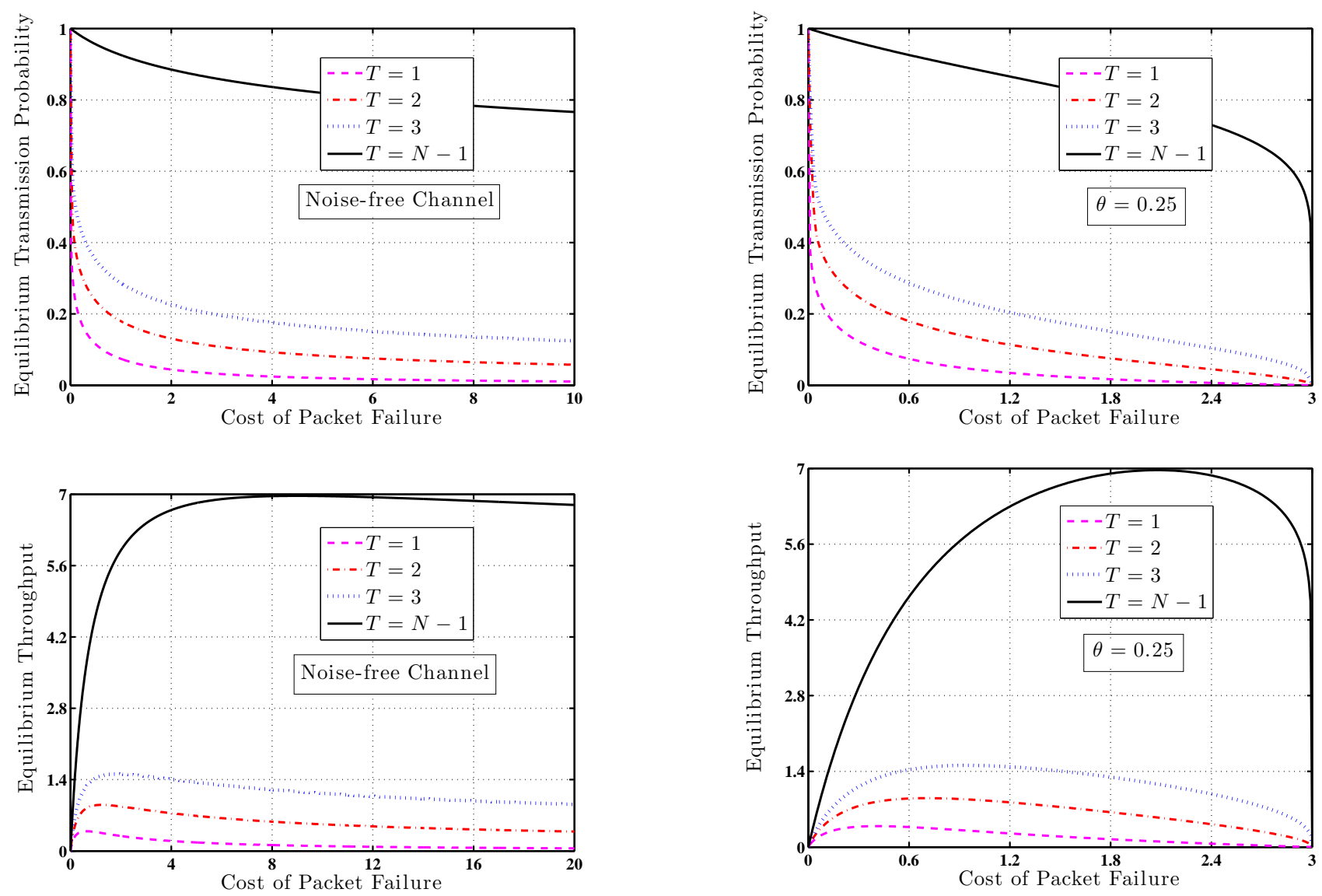

Fig. 4. Equilibrium contention resolution strategies (top figures) and the corresponding system throughput (bottom figures) for $T$-out-of- $N$ channels. Figures on the left are provided for noise-free channels, whereas figures on the right are for the common noise parameter $\theta=0.25$. In all cases, $N=10$. The unit of throughput is normalized to packets per time slot.

packets are destroyed together. If the noise parameter $\theta_{n}$ is 0 for all $n$, then this is the channel model studied in [23] and [24]. Such channels can be implemented by using $T$-out-of- $N$ codes [25].

2) Equilibrium Contention Resolution Strategies: We will focus only on the homogenous case and the Nash equilibrium at which all users access the channel with positive probability for illustrative purposes, but a similar analysis can be conducted for the heterogenous case and other equilibria as in the collision channel model above. We set the strategy sets to $[0,1]$. In this case, $J(p)$ in (1) is given by $J(p)=\sum_{n=0}^{T-1}\left(1-\theta_{n+1}\right)\left(\begin{array}{c}N-1 \\ n\end{array}\right) p^{n}(1-p)^{N-1-n}$. The common equilibrium transmission probability $p^{\star}$ is obtained by solving $J\left(p^{\star}\right)=\frac{c}{1+c}$. If $T<N$, then $J(1)=0$, and it is enough to have $J(0)=1-\theta_{1} \geq \frac{c}{1+c}$ for the existence of $p^{\star}$ solving $J\left(p^{\star}\right)=\frac{c}{1+c}$. Otherwise, $J(1)=1-\theta_{N}$, and we require $1-\theta_{1} \geq \frac{c}{1+c} \geq 1-\theta_{N}$. Note also that if $1-\theta_{N}>\frac{c}{1+c}$ (for $T=N$ ), then users transmit with probability one, and if $1-\theta_{1}<\frac{c}{1+c}$ (for $T \leq N$ ), they never transmit.

3) Numerical Results and Discussion: In Fig. 4, we plot the equilibrium strategies and the corresponding system throughput for noise-free channels and for the channels with the common noise parameter $\theta=0.25$ when $N=10$. Again, the unit of throughput is normalized to packets per time slot. Similar conclusions continue to hold for other values of $N$ and $\theta_{n}$ varying with $n$. As expected, equilibrium transmission probabilities and the corresponding throughput increase with $T$. More importantly, the maximum achievable throughput increases more than linearly with $T$. We find this maximum throughput to be around $0.39,0.9,1.51$ and 6.97 for $T=1,2,3$ and 9 , respectively. For $T$ large when $\theta=0.25$, we also observe a severe cut-off in transmission probabilities and a corresponding sharp decrease in the equilibrium throughput when the cost of packet failure comes close to the critical level $\frac{1-\theta}{\theta}$. On the other hand, throughput does not exhibit such an abrupt decrease with increasing cost for the noisefree channel. This indicates the importance of the calibration of costs and noise levels in order to avoid high penalty in equilibrium throughput for noisy $T$-out-of- $N$ channels with large multipacket reception capability.

\section{Selfish Mobile Users with Pairwise Transmissions}

1) Channel Model: As a final example, we consider pairwise transmissions in which the positions of $N$ transmitterreceiver pairs $\left\{\mathrm{TX}_{i}, \mathrm{RX}_{i}\right\}_{i=1}^{N}$ are independently and identically distributed over a network domain. Transmission from $\mathrm{TX}_{i}$ is successful if there is no other transmitter closer to $\mathrm{RX}_{i}$ than $\mathrm{TX}_{i}$, and the channel noise is small enough, which will be assumed to happen with probability $1-\theta$. Here, the interpretation of $\theta$ is the same as above. This is the first 

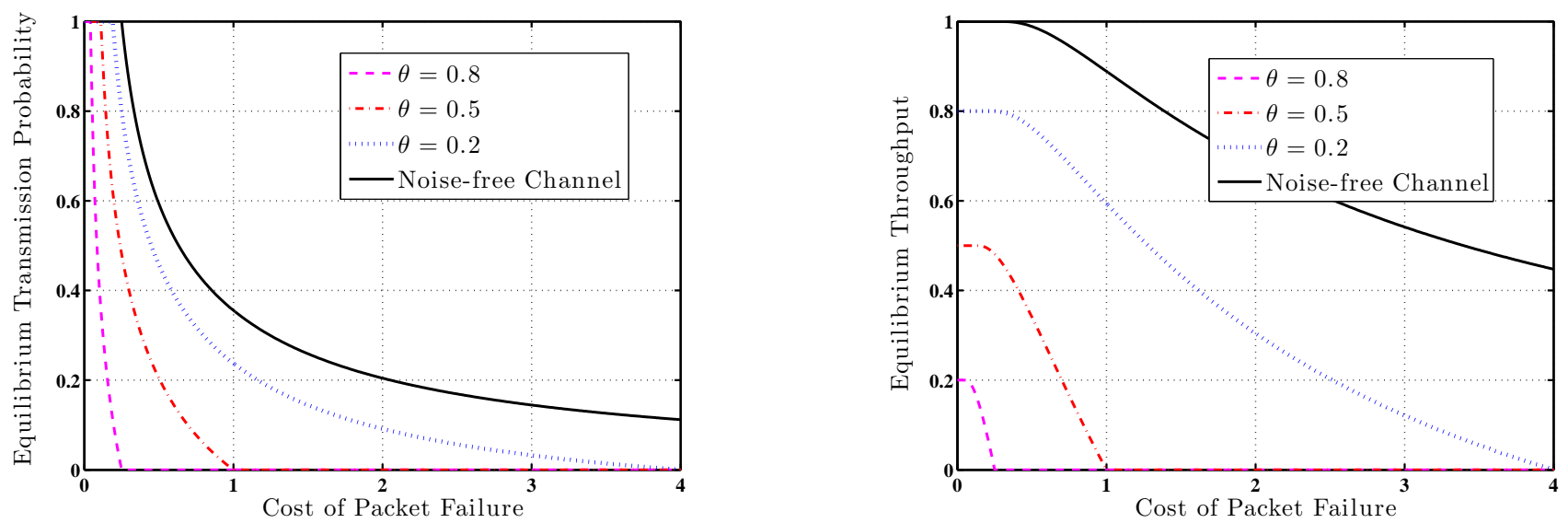

Fig. 5. Equilibrium contention resolution strategies (left figure) and the corresponding system throughput (right figure) for mobile users with pairwise transmissions. In all cases, $N=5$. The unit of throughput is normalized to packets per time slot.

example studied in [9], and shows the generality of our results beyond the simple multiple access channel with one common receiver and multiple transmitters. More applications of our results in Section III are possible, e.g., wireless channels with capture and frequency hopping spread spectrum systems, but we do not pursue this direction due to space limitation.

2) Equilibrium Contention Resolution Strategies: For illustrative purposes, we again focus on the homogenous case and the symmetric equilibrium at which all users access the channel with positive probability. We set the strategy sets to $[0,1]$. For this set-up, we have $\gamma_{n}=\frac{1-\theta}{n}$, and $J(p)=\sum_{n=0}^{N-1} \frac{1-\theta}{n+1}\left(\begin{array}{c}N-1 \\ n\end{array}\right) p^{n}(1-p)^{N-1-n}$. The equilibrium transmission probability is obtained by solving $J\left(p^{\star}\right)=\frac{c}{1+c}$. $p^{\star}$ exists if $J(0)=1-\theta \geq \frac{c}{1+c} \geq J(1)=\frac{1-\theta}{N}$. If $1-\theta<\frac{c}{1+c}$, users never transmit, and if $\frac{1-\theta}{N}>\frac{c}{1+c}$, they transmit with probability one.

3) Numerical Results and Discussion: In Fig. 5, we show equilibrium transmission probabilities and the corresponding throughputs in packets per time slot for different values of $\theta$ when there are $N=5$ transmitter-receiver pairs in the network. Similar conclusions continue to hold for other values of $N$. To avoid repetition, we will mention only the main differences between this scenario and the two previous ones. In the current case, there is an interval of $c$ around zero in which transmission probabilities become one. The length of this interval is determined by $\theta$ and $N$, and is given by $\frac{1-\theta}{N-1+\theta}$. On the other hand, transmission with probability one can arise only in the limit when $c$ goes to zero in Figs. 2 and 4 .

The behavior of the equilibrium throughput in this case looks quite different from the ones in previous examples. This behavior arises because the average number of successful receptions with pairwise transmissions is always equal to $1-\theta$ for any $n$ since $\gamma_{n}=\frac{1-\theta}{n}$. Therefore, when transmission probabilities are low, the network becomes underutilized and the overall network throughput decreases. On the other hand, when transmission probabilities are high, there always are active transmitter-receiver pairs for which the network can support a throughput of $1-\theta$.

\section{Asymptotic Network Behavior}

For the maximum stable throughput analysis in multiple access networks, it is commonly assumed that there is an infinite population of users and the number of packet arrivals from them follows a Poisson law, e.g., see [7], [8] and [11]. In the absence of any knowledge of micro-level rules governing user behavior, such a Poisson assumption for predicting collective behavior of an infinite population of users is very natural to make by the law of rare events [26], [27].

However, having established micro-level contention resolution strategies, we can now verify whether or not the Poisson distribution is still valid to approximate the total number of packet arrivals from an infinite population of selfish users. In fact, it is shown in [15] that such an approximation holds for identical users. In this section, we will establish a similar Poisson-Bernoulli approximation result for heterogenous users in which the cost of packet failure of user $i$ is $c_{i}>0$. In establishing our approximation result, we focus only on noise-free collision channels and the equilibrium at which all users transmit with positive probability. We assume that cost parameters of initial users do not change as we add more users to the system. Extensions to imperfect collision channels and other equilibria with an infinite population of users are trivial. Extension to general channel models is a more challenging future research direction.

The main result of this section is given in Theorem 3. For any given two discrete random variables $X$ and $Y$ taking values in a countable set $\mathcal{Z}, d_{T V}(X, Y)$ represents the total variation distance between their distributions $F_{X}$ and $F_{Y}$, defined as $d_{T V}(X, Y)=\sum_{z \in \mathcal{Z}}\left|F_{X}(z)-F_{Y}(z)\right|$. Theorem 3 indicates that, even though a pure Poisson approximation may not work well, a mixture of a Poisson distribution with finitely many Bernoulli distributions serves as a good approximation for the total number of packet arrivals from an infinite population of heterogenous users.

Theorem 3: Let $\boldsymbol{p}_{N}^{\star}$ be a Nash equilibrium at which all users transmit with positive probability. If $\boldsymbol{p}_{N}^{\star}$ exists for all $N$ large enough, then the total number of packet arrivals $Y_{N}$ at $\boldsymbol{p}_{N}^{\star}$ converges in distribution to a real valued random variable 


$$
\tilde{\Gamma}_{i}\left(\boldsymbol{R}, \boldsymbol{s}_{-i}\right)=\sum_{n=1}^{N} \sum_{\substack{\mathcal{I}_{n} \subseteq \mathcal{I}: \\ i \in \mathcal{I}_{n},\left|\mathcal{I}_{n}\right|=n}} \gamma_{n} \prod_{j \in \mathcal{I}_{n}-\{i\}} \int_{0}^{\infty} s_{j}\left(c_{j}\right) d F_{i, j}\left(c_{j}\right) \prod_{j \in \mathcal{I}-\mathcal{I}_{n}}\left(1-\int_{0}^{\infty} s_{j}\left(c_{j}\right) d F_{i, j}\left(c_{j}\right)\right)
$$

$Y$ if and only if $\lim _{N \rightarrow \infty} \sum_{i=1}^{N} p_{i, N}^{\star} \in(0, \infty)$. Moreover, for any $\epsilon>0$, there exist a Poisson random variable $\operatorname{Po}(\lambda)$ with mean $\lambda$ and an independent finite collection of Bernoulli random variables $\left\{\operatorname{Bern}\left(q_{k}\right)\right\}_{k=1}^{K}$ with means $\left\{q_{k}\right\}_{k=1}^{K}$ such that $d_{T V}\left(Y, \operatorname{Po}(\lambda)+\sum_{k=1}^{K} \operatorname{Bern}\left(q_{k}\right)\right) \leq \epsilon$.

Proof: See Appendix C.

Intuitively, this result can be interpreted as follows. There is a homogenous part of the population consisting of users with similar costs. Packet arrivals from these users behave like a Poisson random process collectively. On the other hand, the rest of the users are more heterogeneous, and the number of packet arrivals from them can be approximated as a summation of Bernoulli random variables. In fact, in our proof in Appendix C, we showed that the existence of a Nash equilibrium with an infinite population of users necessitates costs of packet failures to have only one accumulation point. The Poisson and Bernoulli parameters can be fine tuned to increase the approximation accuracy. Moreover, by applying the Stein-Chen approximation [27] to the Bernoulli part, we can further reduce the Poisson-Bernoulli approximation to a pure Poisson approximation, albeit with greater approximation error.

\section{RANDOM ACCESS WITH IMPERFECT COST INFORMATION}

Our analysis in previous sections depended implicitly on the perfect information assumption in which all users know the costs of all other users perfectly. In this section, we will show how we can relax this perfect information assumption.

\section{A. Imperfect Information Game Formulation and Equilibrium Strategies}

We focus on the random access game in the standard form without loss of generality, and assume that $c_{i}$ is randomly distributed according to a cost distribution $F_{i}$ but is perfectly known by user $i$ before the start of a transmission. On the other hand, user $i$ does not know the cost values of other users exactly but only has a set of belief distributions $\left\{F_{i, j}\right\}_{j \in \mathcal{I}-\{i\}}$ to predict them. This formulation is general enough to admit having belief distributions different than true cost distributions. In this Bayesian game setting, the strategy of user $i$ is a function $s_{i}$ that maps $c_{i} \in(0, \infty)$ to a transmission probability $p_{i} \in\left[\alpha_{i}, \beta_{i}\right]$. With a slight abuse of notation, we will still represent the strategy set of user $i$ by $\mathcal{S}_{i}$. As is standard, a strategy profile $s^{\star}$ is said to be a Nash equilibrium if $s_{i}^{\star}$ is a solution of the selfish utility maximization problem $\max _{s_{i} \in \mathcal{S}_{i}} U_{i}\left(s_{i}, \boldsymbol{s}_{-i}^{\star}\right)$ for all $i \in \mathcal{I}$. In contrast to our analysis in Section III, this optimization problem is now over infinite dimensional function spaces. However, as established in the next theorem, equilibrium strategy profiles can be identified by using a threshold vector $\tau^{\star}$ in $\mathbb{R}_{+}^{N}$.
Theorem 4: Let $\tilde{\Gamma}_{i}\left(\boldsymbol{R}, \boldsymbol{s}_{-i}\right)$ be as in (3). A strategy profile $\boldsymbol{s}^{\star}$ is a Nash equilibrium if and only if $s_{i}^{\star}$ is a threshold strategy in the form $s_{i}^{\star}\left(c_{i}\right)=\beta_{i} 1_{\left\{c_{i}<\tau_{i}^{\star}\right\}}+p_{i} 1_{\left\{c_{i}=\tau_{i}^{\star}\right\}}+\alpha_{i} 1_{\left\{c_{i}>\tau_{i}^{\star}\right\}}$ almost surely (with respect to $F_{i}$ ) for all $i$, where $\tau_{i}^{\star} \stackrel{=}{=}$ $\frac{\tilde{\Gamma}_{i}\left(\boldsymbol{R}, \boldsymbol{s}_{-i}^{\star}\right)}{1-\tilde{\Gamma}_{i}\left(\boldsymbol{R}, \boldsymbol{s}_{-i}^{\star}\right)}$ and $p_{i} \in\left[\alpha_{i}, \beta_{i}\right]$.

Proof: See Appendix D.

\section{B. Existence and Uniqueness of the Nash Equilibria}

There are several important theoretical and practical implications of Theorem 4. First of all, it allows us to restrict the search for equilibrium strategies to only threshold strategies. Therefore, by viewing the random access game as a game in which users choose a threshold, we can write the best-response function $\boldsymbol{B}: \mathbb{R}_{+}^{N} \mapsto \mathbb{R}_{+}^{N}$, with a slight abuse of notation, as $\boldsymbol{B}(\boldsymbol{\tau})=\left(\frac{\tilde{\Gamma}_{1}\left(\boldsymbol{R}, \boldsymbol{\tau}_{-1}\right)}{1-\tilde{\Gamma}_{1}\left(\boldsymbol{R}, \boldsymbol{\tau}_{-1}\right)}, \frac{\tilde{\Gamma}_{2}\left(\boldsymbol{R}, \boldsymbol{\tau}_{-2}\right)}{1-\tilde{\Gamma}_{2}\left(\boldsymbol{R}, \boldsymbol{\tau}_{-2}\right)}, \cdots, \frac{\tilde{\Gamma}_{N}\left(\boldsymbol{R}, \boldsymbol{\tau}_{-N}\right)}{1-\tilde{\Gamma}_{N}\left(\boldsymbol{R}, \boldsymbol{\tau}_{-N}\right)}\right)$. By restricting thresholds to take values only from $[0, \Delta]$ for some large but finite positive constant $\Delta$ and assuming all belief distributions are continuous, we can use the Brouwer fixed point theorem to conclude that $\boldsymbol{B}$ has at least one fixed point, which is the Nash equilibrium of the random access game. Note that searching for equilibrium thresholds in a closed interval is simply a technical requirement to apply the Brouwer fixed point theorem but is not a severe practical limitation. It is always correct that $\tilde{\Gamma}_{i}\left(\boldsymbol{R}, \boldsymbol{\tau}_{-i}^{\star}\right) \leq \tilde{\Gamma}_{i}(\boldsymbol{R}, \mathbf{0})=r_{1,1}$, and therefore as long as the channel is imperfect with $r_{1,1}<1$ due to various random effects such as fading, path-loss and background noise, the best-response threshold of a user never becomes larger than $\frac{r_{1,1}}{1-r_{1,1}}$. The same argument also shows the existence of a symmetric Nash equilibrium for the symmetric game in which all users have the same belief distribution to predict the costs of others. Moreover, by appealing to [28], we can also ensure the uniqueness of the Nash equilibrium if the Jacobian of $\boldsymbol{B}$ does not have an eigenvalue equal to 1 for all $\tau \in[0, \Delta]^{N}$. However, this condition must be checked case-by-case for each channel model, which can be a computationally extensive task.

\section{Best-response Learning and Discussion}

On the practical side, Theorem 4 implies that user $i$ can learn to play its best-response against its opponents' strategies by estimating the congestion signal $\tilde{\Gamma}_{i}\left(\boldsymbol{R}, \boldsymbol{\tau}_{-i}\right)$ even if it fails to predict their strategies perfectly at the beginning of the game. Intuitively, $\tilde{\Gamma}_{i}\left(\boldsymbol{R}, \boldsymbol{\tau}_{-i}\right)$ can be thought of as a summary statistic summarizing the strategies of other users, and its knowledge is sufficient for user $i$ to play its best-response. $\tilde{\Gamma}_{i}\left(\boldsymbol{R}, \boldsymbol{\tau}_{-i}\right)$ can be estimated by counting the number of successful transmissions, or the number of idle slots for collision channels as in [3], which leads to decoupling of contention resolution from handling failed transmissions. Such a learning process on the best-response path, if it converges, 

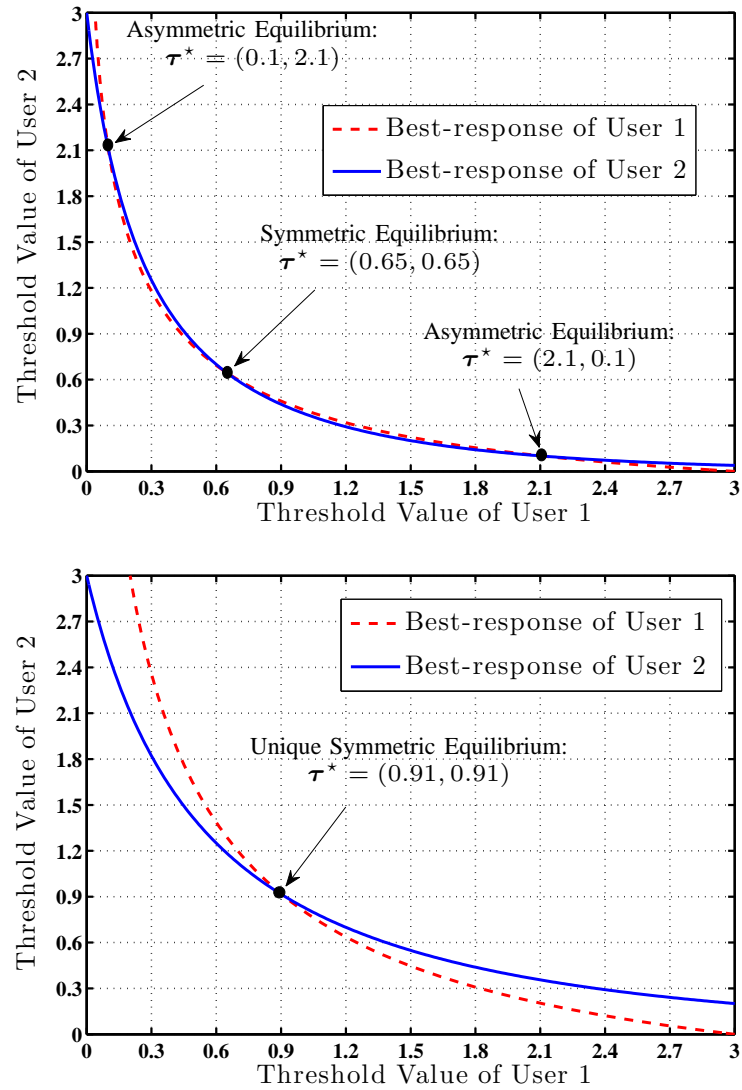
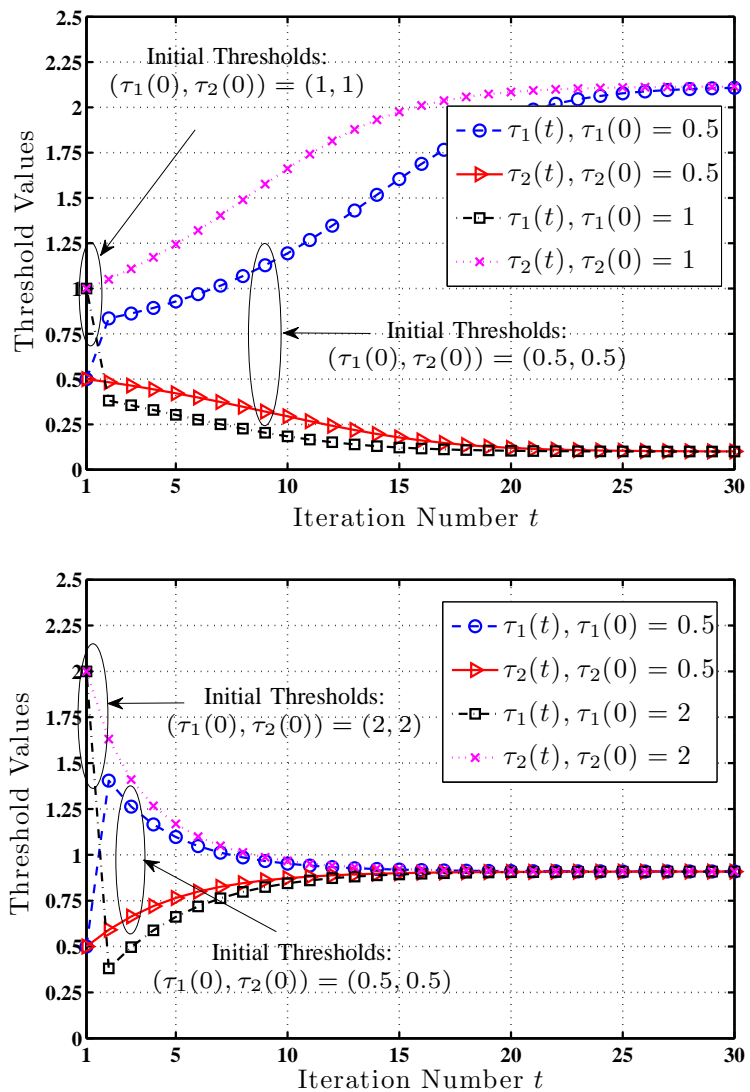

Fig. 6. For the imperfect collision channel with $r_{1,1}=0.75$, the left figures illustrate best-response functions of users 1 and 2 , while the right figures illustrate the learning process leading to Nash equilibria. Belief distributions are exponential with parameters $\lambda=1$ (top figures) and $\lambda=0.5$ (bottom figures).

leads the network to an equilibrium operating point [29], which is what we illustrate next.

In Fig. 6, a numerical application is illustrated for the imperfect collision channel in which two users having the same exponential belief distribution with parameter $\lambda>0$, i.e., $F_{1,2}(c)=F_{2,1}(c)=F(c)=1-\mathrm{e}^{-\lambda c}$, contend for the channel access. We set $r_{1,1}$ to 0.75 .

Even for this simple symmetric two-user case, the resulting network behavior can be quite complicated. To start with, depending on the value of $\lambda$, the equilibrium does not need to be unique or symmetric. For example, in the top left figure in Fig. 6, we show the communication scenario in which $\lambda=1$ and the best-response functions of users intersect at three different points, two of which correspond to asymmetric equilibria at which one user sets its threshold to 0.1 , while the other one sets it to 2.1. Moreover, the symmetric equilibrium is unstable, i.e., small perturbations to this equilibrium will lead the system to converge to an asymmetric equilibrium when there are multiple equilibria. That is, starting from any initial condition except for the symmetric equilibrium, the learning process on the best-response path converges to only one of these asymmetric equilibria. For example, in the top right figure in Fig. 6, we show two learning curves with different initial conditions leading to these asymmetric equilibria. At iteration $t+1$, user 1 moves first and updates its threshold to $\tau_{1}(t+1)=\frac{\tilde{\Gamma}_{1}\left(\boldsymbol{R}, \tau_{2}(t)\right)}{1-\tilde{\Gamma}_{1}\left(\boldsymbol{R}, \tau_{2}(t)\right)}$ by estimating
$\tilde{\Gamma}_{1}\left(\boldsymbol{R}, \tau_{2}(t)\right)$. Then, user 2 moves and updates its threshold to $\tau_{2}(t+1)=\frac{\tilde{\Gamma}_{2}\left(\boldsymbol{R}, \tau_{1}(t+1)\right)}{1-\tilde{\Gamma}_{2}\left(\boldsymbol{R}, \tau_{1}(t+1)\right)}$ by estimating $\tilde{\Gamma}_{2}\left(\boldsymbol{R}, \tau_{1}(t+1)\right)$. Iteration $t+1$ terminates after user 2 updates its threshold. As a result, the time-scale of each iteration corresponds to several tens of time-slots in a physical system allowing users to accurately estimate the congestion signals. Based on these observations, we conclude that the symmetric equilibrium, desirable for fairness purposes, exists but may never appear even in symmetric communication scenarios when there are multiple equilibria.

On the other hand, when there is a unique equilibrium, which is automatically symmetric due to the symmetry in the problem, it is also the stable equilibrium in this case. In the bottom left figure in Fig. 6, we show the scenario in which $\lambda=0.5$ and the best-response functions intersect at the unique equilibrium $\tau^{\star}=(0.91,0.91)$. In the bottom right figure in Fig. 6, we show learning curves with different initial conditions, and leading to the unique symmetric equilibrium. We note that convergence to the equilibrium in both cases of multiple equilibria and unique equilibrium is fast, and only takes around 30 iterations.

Further insight into this behavior can be gained by looking at the two-step-forward best-response function $G$. Let $B_{i}\left(\tau_{-i}\right)$ be the best-response of user $i$ against its opponent's strategy $\tau_{-i}$. Then, $B_{i}\left(\tau_{-i}\right)=\frac{r_{1,1}}{\mathrm{e}^{\lambda \tau-i}-r_{1,1}}$, and $G\left(\tau_{1}\right)=B_{1}\left(B_{2}\left(\tau_{1}\right)\right)$ 


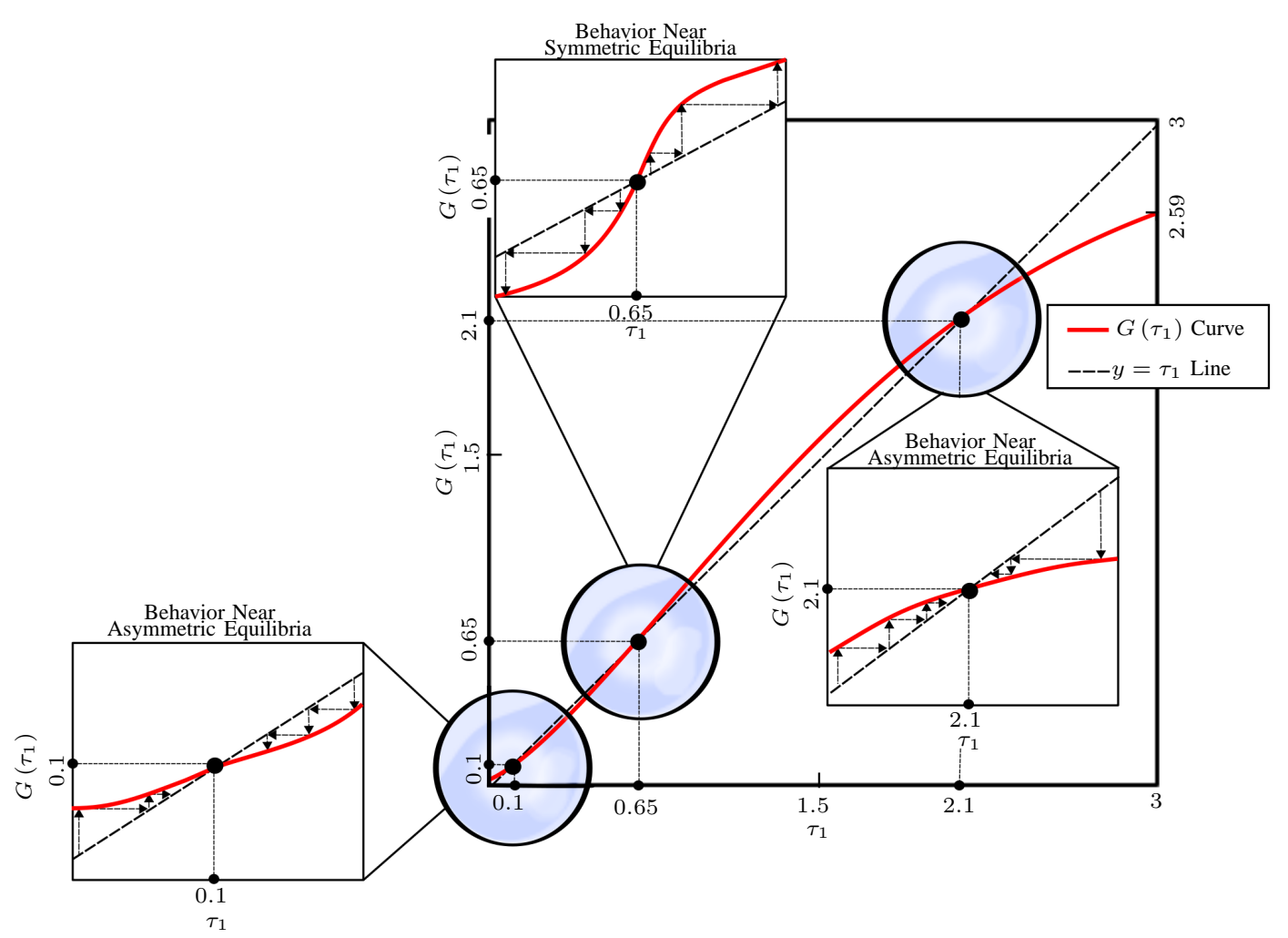

Fig. 7. Best-response learning dynamics around symmetric and asymmetric Nash equilibria for the imperfect collision channel. $N=2, r_{1,1}=0.75$ and $\lambda=1$.

is equal to

$$
G\left(\tau_{1}\right)=\frac{r_{1,1}}{\exp \left(\frac{\lambda r_{1,1}}{\mathrm{e}^{\lambda \tau_{1}-r_{1,1}}}\right)-r_{1,1}} .
$$

Intuitively, $G\left(\tau_{1}\right)$ is the threshold value that user 1 sets after user 2 chooses its best-response against $\tau_{1}$. Therefore, if $G\left(\tau_{1}(t)\right)>\tau_{1}(t)$, then user 1 increases its threshold in the next iteration, whereas it decreases it if $G\left(\tau_{1}(t)\right)<\tau_{1}(t)$. If $G\left(\tau_{1}(t)\right)=\tau_{1}(t)$, then the system is in equilibrium. In Fig. 7 , for $\lambda=1$, we show the behavior of $G$ in $[0,3]$ by further zooming in on its behavior around probable user 1 equilibrium threshold values in the inset figures. If $\tau_{1}$ is anywhere in between 0.1 and 0.65 , even if arbitrarily close to 0.65 , user 1 keeps it decreasing in the next iteration until the system achieves the equilibrium $(0.1,2.1)$. Similarly, it keeps it increasing until the system achieves the equilibrium $(2.1,0.1)$ if $\tau_{1} \in(0.65,2.1)$. Similar explanations hold if $\tau_{1}$ is below 0.1 or above 2.1 . Hence, the symmetric equilibrium is unstable, and only the asymmetric equilibria appear as a result of the best-response learning process. On the other hand, when the equilibrium is unique, similar arguments show that it is stable and all initial threshold pairs are in the domain of attraction of this equilibrium for this case.

Finally, we compare equilibrium contention resolution strategies and the corresponding throughput performance over a noisy collision channel with noise parameter $\theta$ for perfect and imperfect information random access games in Fig. 8. We set $N$ to 2 for simplicity. Recall that $r_{1,0}=\theta$ and $r_{1,1}=1-\theta$. In the imperfect information case, both users have the same exponential belief distribution with parameter $\lambda>0$, i.e., $F_{1,2}(c)=F_{2,1}(c)=F(c)=1-\mathrm{e}^{-\lambda c}$. Furthermore, transmission costs are also randomly generated according to $F(c)$. As explained above, users in the imperfect information random access game set their best-response thresholds to maximize their expected utilities by exploiting the statistical information given by their belief distributions about their opponents, and then decide to transmit or not after observing their realized cost values. For fairness of comparison, transmission probabilities and the corresponding equilibrium throughput are plotted against the mean cost value $\frac{1}{\lambda}$ in the imperfect information random access game, and only the symmetric equilibrium is considered.

Surprisingly, the equilibrium throughput performance achieved under imperfect cost information is larger than that achieved under perfect cost information. In particular, when compared to the perfect information case, users under imperfect cost information contend for the channel access less aggressively as the mean cost value approaches zero, and conversely become more aggressive as the mean cost value grows to infinity. To put it in other words, providing selfish 

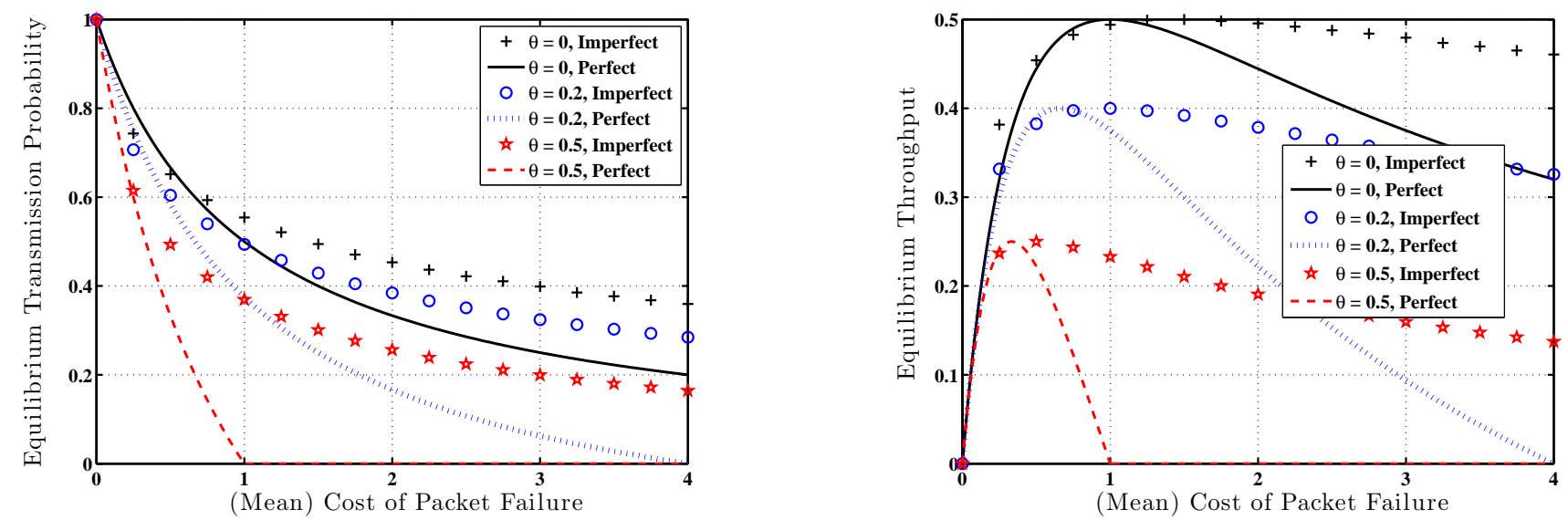

Fig. 8. Comparison of equilibrium contention resolution strategies (left figure) and the corresponding system throughput (right figure) for perfect and imperfect information random access game formulations. Transmission costs are generated according to the exponential distribution $F(c)=1-\mathrm{e}^{-\lambda c}$ in the imperfect information random access game, where $\frac{1}{\lambda}$ is interpreted as the cost of packet failure for this case in the figures. In all cases, $N=2$. The unit of throughput is normalized to packets per time slot.

mobile users with more information about their opponents makes them unnecessarily more risk averse for high values of the transmission cost, and more risk-taking for low values of the transmission cost. Indeed, this phenomenon is similar to the Braess-like paradoxes observed in both the engineering and applied game theory literature [30]-[32], and hints at an important design criterion for next generation wireless random access protocols: Always maintain some level of randomness to better tame selfishness in mobile users for higher target data rates.

\section{Future Research Directions}

Before concluding this section, we mention briefly some future research directions of interest within this Bayesian random access game formulation. One issue of interest is to reveal more comprehensive learning dynamics in more general channel settings. Even though the same principles and techniques can be applied, the system behavior observed for imperfect collision channels does not necessarily extend to other channel models in a straightforward manner. For example, the uniqueness of a Nash equilibrium does not always imply that users can learn to play it. When the collision channel is noise-free, the equilibrium is unique [16] but unstable. Similarly, when the number of users increases, the set of Nash equilibria becomes more complicated to analyze. For arbitrary numbers of users, determining stable equilibria, and identifying initial conditions leading to such equilibria with required performance criteria are other future research problems of interest. Solving these problems could lead to designs for efficient random access control mechanisms, and to reverse/forward engineering [4], [5] of existing ones to enforce desirable system performance.

\section{CONCLUSION}

In this paper, we have focused on layer 2 contention resolution strategies for wireless networks with multipacket reception. The multipacket reception capability is an important feature of our model to capture probabilistic receptions in wireless multiple access systems. We have obtained necessary and sufficient conditions for a Nash equilibrium strategy profile. To demonstrate tangible applications, these equilibrium conditions have been solved for specific channel models to derive contention resolution strategies and to analyze the resulting network performance. Various engineering insights such as designing utility functions to maximize communication rates and to achieve service differentiation have been illustrated based on the derived equilibrium strategies. We have also studied the asymptotic collective equilibrium behavior, and established a Poisson-Bernoulli approximation for the total number of packet arrivals from an infinite selfish user population. Finally, we have examined the contention resolution problem with imperfect information, derived the form of equilibrium strategies, established their existence and uniqueness, and analyzed a strategy update mechanism based on best-response dynamics converging to an equilibrium.

\section{REFERENCES}

[1] R. Bender and G. Sandstrom, "Wireless carriers refine 4G technology," The Wall Street Journal, Available Online: http://online.wsj.com/article/SB10001424052748704869304575109624056 054264.html, Mar 2010.

[2] T. Cui, L. Chen and S. H. Low, "A game-theoretic framework for medium access control," IEEE J. Sel. Areas Commun., vol. 26, no. 7, pp. 1116-1127, Sept 2008.

[3] L. Chen, S. H. Low and J. C. Doyle, "Random access game and medium access control design," IEEE/ACM Trans. Netw., vol. 18, no. 4, Aug 2010.

[4] J.-W. Lee, A. Tang, J. Huang, M. Chiang and A. R. Calderbank, "Reverseengineering MAC: A non-cooperative game model," IEEE J. Sel. Areas Commun., vol. 25, no. 6, pp. 1135-1147, Aug 2007.

[5] J. Barcelo, H. Inaltekin and B. Bellalta, "Obey or play: Asymptotic equivalence of slotted Aloha with a game theoretic contention model," IEEE Commun. Lett., vol. 15, no. 6, pp. 623-625, June 2011.

[6] N. Abramson, "The Aloha system - another alternative for computer communications," in Fall Joint Computer Conf., AFIPS Conf. Proc., vol. 37, pp. 281-285, 1970.

[7] B. Hajek and T. Van Loon, "Decentralized dynamic control of a multiaccess broadcast channel," IEEE Trans. Autom. Control, vol. AC-27, no. 3, pp. 559-569, June 1982.

[8] J. N. Tsitsiklis, "Analysis of a multiaccess control scheme," IEEE Trans. Autom. Control, vol. AC-32, no. 11, pp. 1017-1020, Nov 1987. 
[9] S. Ghez, S. Verdú and S. C. Schwartz, "Stability properties of slotted Aloha with multipacket reception capability," IEEE Trans. Autom. Control, vol. 33, no. 7, pp. 640-649, July 1988.

[10] V. Anantharam, "The stability region of the finite-user slotted Aloha protocol," IEEE Trans. Inf. Theory, vol. 37, no. 3, pp. 535-540, May 1991.

[11] D. Bertsekas and R. Gallager, Data Networks, Prentice-Hall, Upper Saddle River, NJ, second edition, 1992.

[12] Y. Jin and G. Kesidis, "Equilibria of a noncooperative game for heterogenous users of an ALOHA network," IEEE Commun. Lett., vol. 6, no. 7, pp. 282-284, July 2002.

[13] A. B. MacKenzie and S. B. Wicker, "Selfish users in Aloha: A gametheoretic approach," in Proc. IEEE Vehicular Technology Conf., vol. 3, pp. 1354-1357, Atlantic City, NJ, Oct 2001.

[14] A. B. MacKenzie and S. B. Wicker, "Stability of multipacket slotted Aloha with selfish users and perfect information," in Proc. 22nd Annual Joint Conference of the IEEE Computer and Communications Societies, vol. 3, pp. 1583-1590, San Francisco, CA, Apr 2003.

[15] H. Inaltekin and S. B. Wicker, "The analysis of Nash equilibria of the one-shot random access game for wireless networks and the behavior of selfish nodes," IEEE/ACM Trans. on Netw., vol. 16, no. 5, pp. 1094-1107, Oct 2008.

[16] H. Inaltekin and S. B. Wicker, "Random access games: Selfish nodes with incomplete information," in Proc. IEEE Military Communications Conf., Orlando, FL, Oct 2007.

[17] H. Inaltekin and S. B. Wicker, "Random access game over noisy channels with capture effect," in Proc. 10th ACM International Symposium on Modeling, Analysis and Simulation of Wireless and Mobile Systems, Crete Island, Greece, Oct 2007.

[18] M. H. Ngo and V. Krishnamurthy, "Game theoretic cross-layer transmission policies in multipacket reception wireless networks," IEEE Trans. Signal Process., vol. 55, no. 5, pp. 1911-1926, May 2007.

[19] E. Altman, R. E. Azouzi and T. Jimenez, "Slotted Aloha as a game with partial information," Computer Networks, vol. 45, no. 6, pp. 701-713, Aug 2004.

[20] I. Menache and N. Shimkin, "Rate-based equilibria in collision channels with fading," IEEE J. Sel. Areas Commun., vol. 26, no. 7, pp. 1070-1077, Sept 2008.

[21] I. L. Glicksberg, "A further generalization of the Kakutani fixed point theorem with application to Nash equilibrium points," in Proc. American Mathematical Society, vol. 3, no. 1, pp. 170-174, Feb 1952.

[22] D. Fudenberg and J. Tirole, Game Theory, MIT Press, Cambridge, MA, 1991.

[23] N. Mahravari, "Random-access communication with multiple reception," IEEE Trans. Inf. Theory, vol. 36, no. 3, pp. 614-622, May 1990.

[24] B. S. Tsybakov, V. A. Mikhailov and N. B. Likhanov, "Bounds for packet transmission rate in a random access system," Problemy Peredachi Informatsii, vol. 19, no. 1, pp. 61-81, 1983.

[25] P. Mathys, "A class of codes for a $T$ active users out of $N$ multipleaccess communication system," IEEE Trans. Inf. Theory, vol. 36, no. 6, pp. 1206-1219, Nov 1990.

[26] P. Billingsley, Probability and Measure, John Wiley and Sons, Inc., New York, third edition, 1995.

[27] A. D. Barbour, L. Holst and S. Janson, Poisson Approximation, Oxford University Press, New York, 1992.

[28] R. B. Kellogg, "Uniqueness in the Schauder fixed point theorem," in Proc. American Mathematical Society, vol. 60, no. 1, pp. 207-210, Oct 1976.

[29] D. Fudenberg and D. K. Levine, The Theory of Learning in Games, The MIT Press, Cambridge, MA, 1998.

[30] Y. A. Korilis, A. A. Lazar and A. Orda, "Avoiding the Braess paradox in non-ccoperative networks," J. Appl. Prob., vol. 36, no. 1, pp. 211-222, Mar 1999.

[31] H. Kameda, E. Altman, T. Kozawa and Y. Hosokawa, "Braess-like paradoxes in distributed computer systems," IEEE Trans. Autom. Control, vol. 45 , no. 9, pp. 1687-1691, Sept 2000 .

[32] F.-T. Hsu and H.-J. Su, "Random access game in fading channels with capture: Equilibria and Braess-like paradoxes," IEEE Trans. Signal Process., vol. 59, no. 3, pp. 1158-1169, Mar 2011.

\section{APPENDIX A \\ PROOF OF THEOREM 1}

We will first obtain an explicit representation for expected user utilities. Let $\Gamma_{i}\left(\boldsymbol{R}, \boldsymbol{p}_{-i}\right)$ be the probability that a packet transmission from user $i$ becomes successful given the channel reception matrix $\boldsymbol{R}$ and other users' transmission probabilities $\boldsymbol{p}_{-i} . \quad \Gamma_{i}\left(\boldsymbol{R}, \boldsymbol{p}_{-i}\right) \quad$ can be written as $\Gamma_{i}\left(\boldsymbol{R}, \boldsymbol{p}_{-i}\right)=$

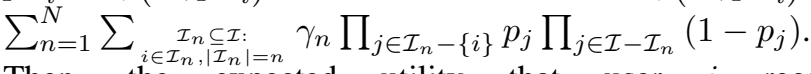

Then, the expected utility that user $i$ receives when the random access game is played according to $\boldsymbol{p}$ is equal to $U_{i}\left(p_{i}, \boldsymbol{p}_{-i}\right)=$ $p_{i}\left(\left(u_{i, S}-u_{i, F}\right) \Gamma_{i}\left(\boldsymbol{R}, \boldsymbol{p}_{-i}\right)-\left(u_{i, W}-u_{i, F}\right)\right)+u_{i, W}$. Now, suppose that $\boldsymbol{p}^{\star}$ is a Nash equilibrium. Firstly, $p_{i}^{\star}$ must be $\beta_{i}$ if $\Gamma_{i}\left(\boldsymbol{R}, \boldsymbol{p}_{-i}\right)>\frac{u_{i, W}-u_{i, F}}{u_{i_{,} S}-u_{i, F}}$ since $U_{i}\left(p_{i}, \boldsymbol{p}_{-i}^{\star}\right)$ is a linear function of $p_{i}$ when $\boldsymbol{p}_{-i}^{\star}$ is fixed. Secondly, $p_{i}^{\star}$ must be $\alpha_{i}$ if $\Gamma_{i}\left(\boldsymbol{R}, \boldsymbol{p}_{-i}\right)<\frac{u_{i, W}-u_{i, F}}{u_{i, S}-u_{i, F}}$. Finally, $p_{i}^{\star}$ can be set to any value in $\left[\alpha_{i}, \beta_{i}\right]$ if $\Gamma_{i}\left(\boldsymbol{R}, \boldsymbol{p}_{-i}\right)=\frac{u_{i, W}-u_{i, F}}{u_{i, S}-u_{i, F}}$. This completes the proof for the only if part of the theorem. The sufficiency of the above equilibrium conditions also follows from similar arguments.

\section{APPENDIX B PRoOF OF THEOREM 2}

Let $\mathcal{I}_{-\{i, j\}}=\mathcal{I}-\{i, j\}$ and $\boldsymbol{p}_{-\{i, j\}}=\left(p_{k}\right)_{k \in \mathcal{I}_{-\{i, j\}}}$. Further, let $\Gamma_{i}\left(\boldsymbol{R}, \boldsymbol{p}_{-i}\right)$ be defined as in the proof of Theorem 1. We can expand $\Gamma_{i}\left(\boldsymbol{R}, \boldsymbol{p}_{-i}\right)$ as a function of $p_{j}$ as

$$
\begin{aligned}
& \Gamma_{i}\left(\boldsymbol{R}, \boldsymbol{p}_{-i}\right)=\Gamma_{i}\left(\boldsymbol{R}, p_{j}, \boldsymbol{p}_{-\{i, j\}}\right) \\
& =p_{j} \sum_{n=0}^{N-2} \sum_{\substack{\mathcal{I}_{n} \subseteq \mathcal{I}_{-\{i, j\}}: \\
\left|\mathcal{I}_{n}\right|=n}} \gamma_{n+2} \prod_{k \in \mathcal{I}_{n}} p_{k} \prod_{k \in \mathcal{I}_{-\{i, j\}}-\mathcal{I}_{n}}\left(1-p_{k}\right) \\
& +\left(1-p_{j}\right) \sum_{n=0}^{N-2} \sum_{\substack{\mathcal{I}_{n} \subseteq \mathcal{I}_{-\{i, j\}}: \\
\left|\mathcal{I}_{n}\right|=n}} \gamma_{n+1} \prod_{k \in \mathcal{I}_{n}} p_{k} \prod_{k \in \mathcal{I}_{-\{i, j\}}-\mathcal{I}_{n}}\left(1-p_{k}\right) .
\end{aligned}
$$

The last equation implies the relation $\Gamma_{i}\left(\boldsymbol{R}, p_{i}, \boldsymbol{p}_{-\{i, j\}}\right)=$ $\Gamma_{j}\left(\boldsymbol{R}, \boldsymbol{p}_{-j}\right)$. We also have $\frac{\partial \Gamma_{i}\left(\boldsymbol{R}, \boldsymbol{p}_{-i}\right)}{\partial p_{j}}<0$, i.e., see (4).

Thus, $\Gamma_{i}\left(\boldsymbol{R}, p_{j}, \boldsymbol{p}_{-\{i, j\}}\right)$ is strictly decreasing in $p_{j}$ for any given fixed $\boldsymbol{p}_{-\{i, j\}}$. Let $\boldsymbol{p}^{\star}$ be a Nash equilibrium such that there exist $p_{i}^{\star}$ and $p_{j}^{\star}$ in $(\alpha, \beta)$ and $p_{i}^{\star} \neq p_{j}^{\star}$. By Theorem 1, this can happen only if $\Gamma_{i}\left(\boldsymbol{R}, p_{j}, \boldsymbol{p}_{-\{i, j\}}^{\star}\right)$ crosses $\frac{c}{1+c}$ at two points $p_{j}^{\star}$ and $p_{i}^{\star}$; but this contradicts the strictly decreasing nature of $\Gamma_{i}\left(\boldsymbol{R}, p_{j}, \boldsymbol{p}_{-\{i, j\}}^{\star}\right)$ as a function of $p_{j}$. Equation 1 follows after some simplifications.

\section{APPENDIX C \\ Proof of THEOREM 3}

We will first obtain an important lemma showing that the existence of a Nash equilibrium with an infinite population of users having positive transmission probabilities necessitates a kind of homogeneity in this population.

Lemma 1: Let $\xi_{i}=\frac{c_{i}}{1+c_{i}}$ and $\xi=\inf _{i \geq 1} \xi_{i}$. If $\boldsymbol{p}_{N}^{\star}$ exists for all $N$ large enough, then $\lim _{i \rightarrow \infty} \xi_{i}=\xi \in(0,1)$.

Proof: Let $\xi(N)=\min _{1 \leq i \leq N} \xi_{i}$. By using Theorem 1 (see also our discussion in Section IV for collision channels), we can write the equilibrium transmission probabilities 


$$
\frac{\partial \Gamma_{i}\left(\boldsymbol{R}, \boldsymbol{p}_{-i}\right)}{\partial p_{j}}=-\sum_{n=0}^{N-2} \sum_{\substack{\mathcal{I}_{n} \subseteq \mathcal{I}_{-\{i, j\}}: \\\left|\mathcal{I}_{n}\right|=n}}\left(\gamma_{n+1}-\gamma_{n+2}\right) \prod_{k \in \mathcal{I}_{n}} p_{k} \prod_{k \in \mathcal{I}_{-\{i, j\}}-\mathcal{I}_{n}}\left(1-p_{k}\right)<0
$$

$\left\{p_{i, N}^{\star}\right\}_{i \in \mathcal{I}}$ as

$$
p_{i, N}^{\star}=1-\xi_{i}^{-1}\left(\prod_{j=1}^{N} \xi_{j}\right)^{\frac{1}{N-1}}
$$

for all $i \in \mathcal{I}$. Hence, existence of $\boldsymbol{p}_{N}^{\star}$ requires $\xi(N) \geq$ $\left(\prod_{i=1}^{N} \xi_{i}\right)^{\frac{1}{N-1}}$. Otherwise, there exists at least one user with an infeasible probability assignment. We first show that $\xi$ is a limit point of $\left\{\xi_{i}\right\}_{i \geq 1}$. If not, for all $\epsilon>0$, there exists a sufficiently large $M$ such that $\xi_{i} \geq \xi+\epsilon$ for all $i \geq M$. This implies $\left(\prod_{i=1}^{N} \xi_{i}\right)^{\frac{1}{N-1}} \geq\left(\prod_{i=1}^{M} \xi_{i}\right)^{\frac{1}{N-1}}(\xi+\epsilon)^{\frac{N-M}{N-1}}$. Thus, $\left(\prod_{i=1}^{N} \xi_{i}\right)^{\frac{1}{N-1}}$ becomes larger than $\xi+\frac{\epsilon}{2}$, and therefore larger than $\xi(N)$, for all sufficiently large $N$, which contradicts the existence of $\boldsymbol{p}_{N}^{\star}$. A similar contradiction also shows that $\xi \in(0,1)$.

Similarly, we can show that $\xi$ is the only limit point of $\left\{\xi_{i}\right\}_{i>1}$. Otherwise, let $\zeta$ be another limit point for $\left\{\xi_{i}\right\}_{i \geq 1}$, and $g(N)$ be the function showing the number of $\xi_{i}$ 's belonging to $(\zeta-\epsilon, \zeta+\epsilon)$ for $1 \leq i \leq N$, and some $\epsilon>0$ small enough satisfying $\zeta-2 \epsilon>\xi+\epsilon$. Since $\zeta$ is a limit point for $\left\{\xi_{i}\right\}_{i \geq 1}, g(N)$ grows to infinity. Observe that $\xi(N) \in[\xi, \xi+\epsilon)$ for all sufficiently large $N$. Then,

$$
\begin{aligned}
\left(\prod_{i=1}^{N} \xi_{i}\right)^{\frac{1}{N-1}} & \geq \xi(N)^{\frac{N-g(N)}{N-1}}(\xi(N)+\epsilon)^{\frac{g(N)}{N-1}} \\
& =\xi(N) \cdot \xi(N)^{\frac{1}{N-1}}\left(1+\frac{\epsilon}{\xi(N)}\right)^{\frac{g(N)}{N-1}} .
\end{aligned}
$$

It is enough to show that $\xi(N)^{\frac{1}{N-1}}=1-O\left(\frac{1}{N}\right)$ and $\left(1+\frac{\epsilon}{\xi(N)}\right)^{\frac{g(N)}{N-1}}=1+O\left(\frac{g(N)}{N}\right)$ to complete the proof. Since the proofs are similar, we will focus only on the proof for the relation $\left(1+\frac{\epsilon}{\xi(N)}\right)^{\frac{g(N)}{N-1}}=1+O\left(\frac{g(N)}{N}\right)$. To this end, the following chain of equalities and inequalities can be obtained by using the Taylor series expansion for the logarithm and the fact that $g(N) \leq N-1$ for all sufficiently large $N$ :

$$
\begin{aligned}
& \left|1-\left(1+\frac{\epsilon}{\xi(N)}\right)^{\frac{g(N)}{N-1}}\right| \\
& =\left|1-\exp \left(\frac{g(N)}{N-1} \log \left(1+\frac{\epsilon}{\xi(N)}\right)\right)\right| \\
& =\frac{g(N)}{N-1} \log \left(1+\frac{\epsilon}{\xi(N)}\right) \cdot \mid 1+\frac{1}{2 !} \frac{g(N)}{N-1} \log \left(1+\frac{\epsilon}{\xi(N)}\right) \\
& \leq \frac{g(N)}{N-1} \log \left(1+\frac{\epsilon}{3 !}\left(\frac{g(N)}{N-1}\right)^{2} \log \left(1+\frac{\epsilon}{\xi(N)}\right)^{2}+\cdots \mid\right.
\end{aligned}
$$

$$
\begin{aligned}
& +\frac{1}{2 !}\left(\frac{g(N)}{N-1}\right)^{2} \log \left(1+\frac{\epsilon}{\xi(N)}\right)^{2}+\cdots \\
\leq & \frac{g(N)}{N-1} \log \left(1+\frac{\epsilon}{\xi(N)}\right) \exp \left(\log \left(1+\frac{\epsilon}{\xi(N)}\right)\right) \\
\leq & \frac{g(N)}{N-1}\left(1+\frac{\epsilon}{\xi}\right) \log \left(1+\frac{\epsilon}{\xi}\right) .
\end{aligned}
$$

This implies that

$$
\begin{aligned}
\left(\prod_{i=1}^{N} \xi_{i}\right)^{\frac{1}{N-1}} & \\
& \geq \xi(N)\left(1+O\left(\frac{g(N)}{N}\right)-O\left(\frac{1}{N}\right)-O\left(\frac{g(N)}{N^{2}}\right)\right) \\
& >\xi(N)
\end{aligned}
$$

for all sufficiently large $N$, which is another contradiction. Hence, $\xi$ is the only limit point of $\left\{\xi_{i}\right\}_{i \geq 1}$, which concludes the proof of the lemma.

We will now prove the if direction. Suppose $\lim _{N \rightarrow \infty} \sum_{i=1}^{N} p_{i, N}^{\star}=m \in(0, \infty)$ exists. Let $F_{N}$ be the distribution of $Y_{N}$. Then, by the Markov inequality, $\operatorname{Pr}\left\{Y_{N}>M\right\} \leq \frac{\mathrm{E}\left[\left(Y_{N}-\mathrm{E}\left[Y_{N}\right]\right)^{2}\right]}{\left(M-\mathrm{E}\left[Y_{N}\right]\right)^{2}}=O\left(\frac{1}{M^{2}}\right)$. Thus, $\left\{F_{N}\right\}_{N \geq 2}$ is a tight sequence of distributions. By using this tightness result, we will now show that $F_{N}$ converges, in variational distance, to a probability distribution $F$. Let $p_{i, \infty}^{\star}=\lim _{N \rightarrow \infty} p_{i, N}^{\star}$, which can be shown to exist by using the above lemma. Then, we also have $\lim _{i \rightarrow \infty} p_{i, \infty}^{\star}=0$. Thus, for any given $\epsilon>0$, we can choose $K$ large enough so that $\max _{i \geq K} p_{i, \infty}^{\star} \leq \frac{\epsilon}{2 m}$. Let $\lambda_{N}=\sum_{i=K}^{N} p_{i, N}^{\star}$ and $\lambda=\lim _{N \rightarrow \infty} \lambda_{N}$. Then, by using metric properties of total variation distance and Le Cam's inequality, we can bound

$$
d_{T V}\left(Y_{N}, \operatorname{Po}(\lambda)+\sum_{i=1}^{K-1} \operatorname{Bern}\left(p_{i, \infty}^{\star}\right)\right)
$$

as $\lim \sup _{N \rightarrow \infty} d_{T V}\left(Y_{N}, \operatorname{Po}(\lambda)+\sum_{i=1}^{K-1} \operatorname{Bern}\left(p_{i, \infty}^{\star}\right)\right) \leq$ $2 \lambda \max _{i \geq K} p_{i, \infty}^{\star} \leq \epsilon$. This implies that $\left\{F_{N}\right\}_{N \geq 2}$ is a Cauchy sequence with respect to the metric $d_{T V}$, and therefore, by using the tightness result above, a limiting probability distribution $F$ to which $F_{N}$ converges exists. This also establishes the Poisson-Bernoulli approximation in Theorem 3.

Now, we prove the only if part. In fact, this will be a general result for any sequence of triangular arrays of Bernoulli random variables. Suppose now $Y_{N}$ converges in distribution to a real valued $Y$. Let $m_{N}=\mathrm{E}\left[Y_{N}\right]$ and $X_{i, N}=Y_{i, N}-p_{i, N}$, where $Y_{i, N}$ is the Bernoulli random variable with mean $p_{i, N}$. First, assume $\lim \sup _{N \rightarrow \infty} m_{N}=\infty$. By using Hölder's inequality, it can be shown that there exists a $\delta_{1}>0$, which is the same for all $p_{i, N}$, such that we have $\mathrm{E}\left[\mathrm{e}^{-t X_{i, N}}\right] \leq 1+t^{2} p_{i, N}$ for all $t \in\left(0, \delta_{1}\right)$. By using this result and Markov's inequality, we can further show that $\operatorname{Pr}\left\{Y_{N} \leq \frac{m_{N}}{2}\right\} \leq \mathrm{e}^{-\frac{t}{4} \sum_{i=1}^{N} p_{i, N}}$ 
for all $t \in\left(0, \delta_{1}\right)$. Since $\lim \sup _{N \rightarrow \infty} m_{N}=\infty$, we can find a subsequence $\left\{m_{N_{k}}\right\}_{k=1}^{\infty}$ such that $m_{N_{k}} \geq k$. Then, $\operatorname{Pr}\left\{Y_{N_{k}} \leq \frac{m_{N_{k}}}{2}\right\} \leq \mathrm{e}^{\frac{-t k}{4}}$. By the Borel-Cantelli lemma, this implies $Y_{N_{k}}$ diverges to $\infty$ almost surely, which is a contradiction. Thus, we must have $\lim \sup _{N \rightarrow \infty} m_{N}<\infty$. This implies uniform integrability of $Y_{N}$. Since the convergence in distribution of uniformly integrable random variables also implies the convergence in mean [26], we have $\lim _{N \rightarrow \infty} \mathrm{E}\left[Y_{N}\right]=\mathrm{E}[Y]<\infty$.

\section{APPENDIX D \\ PROOF OF THEOREM 4}

Assume $s^{\star}$ is a Nash equilibrium. It is easy to see that $\tilde{\Gamma}_{i}\left(\boldsymbol{R}, s_{-i}^{\star}\right)$ is the conditional success probability of user $i$ given that it transmits and others choose their contention resolution strategies according to $s_{-i}^{\star}$. Then, by fixing $s_{-i}^{\star}$ and varying $s_{i}$, the expected utility of user $i$ can be written as

$$
\begin{aligned}
& U\left(s_{i}, \boldsymbol{s}_{-i}^{\star}\right) \\
& =\int_{0}^{\infty} s_{i}\left(c_{i}\right)\left(\tilde{\Gamma}_{i}\left(\boldsymbol{R}, \boldsymbol{s}_{-i}^{\star}\right)-c_{i}\left(1-\tilde{\Gamma}_{i}\left(\boldsymbol{R}, \boldsymbol{s}_{-i}^{\star}\right)\right)\right) d F_{i}\left(c_{i}\right),
\end{aligned}
$$

which is maximized when $s_{i}\left(c_{i}\right)=\beta_{i} 1_{\left\{c_{i}<\tau_{i}^{\star}\right\}}+p_{i} 1_{\left\{c_{i}=\tau_{i}^{\star}\right\}}+$ $\alpha_{i} 1_{\left\{c_{i}>\tau_{i}^{\star}\right\}}$ almost surely. Similarly, if, for all $i \in \mathcal{I}, s_{i}^{\star}$ is in the form $s_{i}^{\star}\left(c_{i}\right)=\beta_{i} 1_{\left\{c_{i}<\tau_{i}^{\star}\right\}}+p_{i} 1_{\left\{c_{i}=\tau_{i}^{\star}\right\}}+\alpha_{i} 1_{\left\{c_{i}>\tau_{i}^{\star}\right\}}$, expected utilities of all users are maximized, and therefore $s^{\star}$ is a Nash equilibrium.

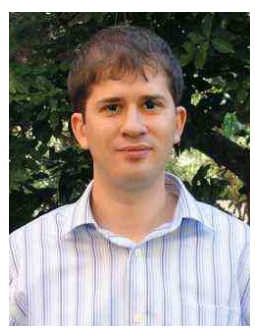

Hazer Inaltekin (S'04, M'06) is an Assistant Professor in the Department of Electrical and Electronics Engineering at Antalya International University, Antalya, Turkey. He received his B.S. degree with High Honors in electrical and electronics engineering from Bogazici University, Istanbul, Turkey, in 2001. He received M.S. and Ph.D. degrees in electrical and computer engineering from Cornell University in 2005 and 2006, respectively. He was with the School of Electrical and Computer Engineering at Cornell University, and then with the Department of Electrical Engineering at Princeton University as a postdoctoral research associate during 2006-2007 and 2007-2009, respectively. In 2009, he joined the Department of Electrical and Electronic Engineering at the University of Melbourne as a research fellow, and was a senior research fellow at the same department between January 2011 and August 2011. His research interests include wireless communications, wireless networks, social networks, game theory, and information theory.

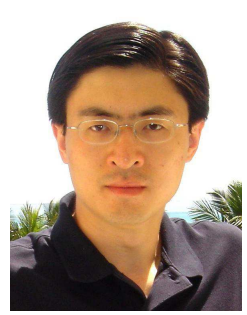

Mung Chiang (S00, M03, SM08) is a Professor of Electrical Engineering at Princeton University, and an affiliated faculty in Applied and Computational Mathematics, and in Computer Science. He received his B.S. (Hons.), M.S., and Ph.D. degrees from Stanford University in 1999, 2000, and 2003, respectively, and was an Assistant Professor 2003-2008 and an Associate Professor 2008-2011 at Princeton University. Chiang's research in networking received awards such as the IEEE Tomiyasu Award, PECASE, TR35, ONR YIP, NSF CAREER, Princeton Wentz Faculty Award, and several best paper awards. His inventions resulted in several technology transfers to commercial adoption, and he founded the Princeton EDGE Lab in 2009. He is currently writing an undergraduate textbook "20 Questions About the Networked Life".

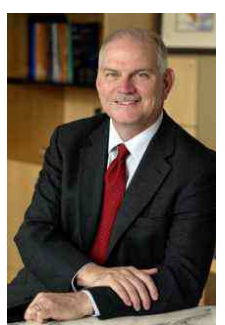

H. Vincent Poor (S'72, M'77, SM'82, F'87) received the Ph.D. degree in EECS from Princeton University in 1977. From 1977 until 1990, he was on the faculty of the University of Illinois at UrbanaChampaign. Since 1990 he has been on the faculty at Princeton, where he is the Michael Henry Strater University Professor of Electrical Engineering and Dean of the School of Engineering and Applied Science. Dr. Poor's research interests are in the areas of stochastic analysis, statistical signal processing and information theory, and their applications in wireless networks and related fields. Among his publications in these areas are the recent books Quickest Detection (Cambridge University Press, 2009) and Information Theoretic Security (Now Publishers, 2009).

Dr. Poor is a member of the National Academy of Engineering and the National Academy of Sciences, a Fellow of the American Academy of Arts and Sciences, and an International Fellow of the Royal Academy of Engineering (U. K). He is also a Fellow of the Institute of Mathematical Statistics, the Optical Society of America, and other organizations. In 1990, he served as President of the IEEE Information Theory Society, and in 2004-07 he served as the Editor-in-Chief of the IEEE Transactions on Information Theory. He received a Guggenheim Fellowship in 2002 and the IEEE Education Medal in 2005. Recent recognition of his work includes the 2009 Edwin Howard Armstrong Achievement Award of the IEEE Communications Society, the 2010 IET Ambrose Fleming Medal for Achievement in Communications, the 2011 IEEE Eric E. Sumner Award, and an honorary D.Sc. from the University of Edinburgh, awarded in June 2011.

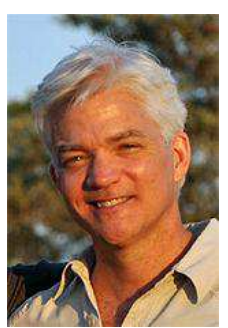

Stephen B. Wicker (Fellow, IEEE) is a Professor of Electrical and Computer Engineering at Cornell University, Ithaca, NY, and a member of the graduate fields of Computer Science, Information Science, and Applied Mathematics. He teaches and conducts research in wireless information networks, cellular networks, packet-switched computer networks, and digital telephony. He currently focuses on the interface between information networking technology, law, and sociology, with a particular emphasis on how design choices and regulation can affect the privacy and speech rights of users.

Professor Wicker was awarded the 1988 Cornell College of Engineering Michael Tien Teaching Award, the 2000 Cornell School of Electrical and Computer Engineering Teaching Award, and the 2009 Cornell University College of Engineering Teaching Award. As of mid 2011, he has supervised 40 doctoral dissertations. Professor Wicker is the author of Codes, Graphs, and Iterative Decoding (Kluwer, 2002), Turbo Coding (Kluwer, 1999), Error Control Systems for Digital Communication and Storage (Prentice Hall, 1995) and Reed-Solomon Codes and Their Applications (IEEE Press, 1994). He has served as Associate Editor for Coding Theory and Techniques for the IEEE Transactions on Communications, and is currently Associate Editor for the ACM Transactions on Sensor Networks. He has served two terms as a member of the Board of Governors of the IEEE Information Theory Society. Professor Wicker is the Cornell Principal Investigator for the TRUST Science and Technology Center - a National Science Foundation center dedicated to the development of technologies for securing the nation's critical infrastructure. 